Wolf Schäfer

\title{
Plutoniumbombe und zivile Atomkraft
}

Carl Friedrich von Weizsäckers Beiträge zum Dritten Reich und zur Bundesrepublik ${ }^{1}$

»Im Grunde wäre ich am allerzufriedensten gewesen, wenn man mich nach 1946 überhaupt nicht mehr gefragt hätte, was wir früher getan haben, sondern nur, was wir künftig tun wollen. Warum soll ich unablässig über die Vergangenheit reden? Ich habe gesagt, was ich zu sagen habe. Es kann sein, daß ich irgendwo bewußt gelogen habe, es kann sein, daß ich verdrängt und unbewußt gelogen habe. Ich bin kein vollkommener Mensch. «2

Carl Friedrich von Weizsäcker, 3. Juni 1993

\section{Einleitung: Der unvollendete »Kreisgang «}

Das Ziel, dem Deutschen Reich die militärische Nutzung der Kernenergie zu ermöglichen, wurde nicht erreicht, aber die Beschränkung auf die friedliche Nutzung der Atomkraft im westdeutschen Nachfolgestaat gelang. Als Initiator, Hauptverfasser und Mitunterzeichner des Göttinger Manifests - der Erklärung von 18 führenden Atomwissenschaftlern, sich nicht "an der Herstellung, der Erprobung oder dem Einsatz von Atomwaffen in irgendeiner Weise zu beteiligen « ${ }^{3}-$ trug von Weizsäcker wesentlich dazu bei, die Weichen der jungen Bundesrepublik auf nichtatomare Bewaffnung zu stellen. ${ }^{4}$

Der historische Lernprozess von Weizsäckers, der 1939 mit dem geheimen Einsatz für den Bau einer deutschen Atombombe begann und 1957 mit dem öffentlichen Widerstand gegen die Aus- und Aufrüstung der Bundesrepublik mit taktischen Atomwaffen endete, hat ungefähr 18 Jahre gedauert. Eine erste, nichtreflexive Phase von sechs Jahren ergibt sich, wenn man, was ich vorschlage, von Weizsäckers Feststellung von 1945 über die angeblich »friedliche Entwicklung der Uranmaschine

1 Für konstruktive Kritik und wertvolle Hinweise danke ich Reinhard Blomert und den anonymen Gutachtern des Leviathan sowie den Teilnehmern des Starnberger Symposiums zum 100. Geburtstag Carl Friedrich von Weizsäckers (veranstaltet am 30. Juni und 1. Juli 2012 von den ehemaligen Mitarbeitern des Max-Planck-Instituts zur Erforschung der Lebensbedingungen der wissenschaftlich-technischen Welt).

2 Weizsäcker 1993, S. 350.

3 Weizsäcker 1988, S. 385; Weizsäcker et al. 1957.

4 Müller 1990, S. 553-558; Kraus 2001, S. 47-67, 187-347; Carson 2010, S. 320 ff. 
[...] unter dem Hitler-Regime « 5 als den Übergangspunkt zur zweiten, reflexiven Phase begreift. Ich betrachte den Weg durch diese zwei Phasen als den von Weizsäcker'schen »Kreisgang «.

Von Weizsäcker war es leid, immer wieder auf das, was er früher getan hatte, angesprochen zu werden. Diesen Aspekt bringt sein Ausspruch, der über diesem Beitrag steht, zum Ausdruck. Er macht deutlich, wie wenig von Weizsäcker daran interessiert war, seinen persönlichen Lernprozess zum Gegenstand öffentlicher Auseinandersetzung zu machen, und wie sehr er wünschte, die Welt würde sich nur mit den Resultaten seines Um- und Nachdenkens beschäftigen. ${ }^{6}$ Das heißt nicht, dass von Weizsäcker sich selbst nachsichtig und unkritisch betrachtete, vermutlich ganz im Gegenteil. ${ }^{7}$ Aber von dem jüngeren Mann, der er einmal war und der auf der besiegten Hitlerseite gestanden hatte, setzte er sich schweigend ab. Daraus folgte, dass der ältere von Weizsäcker so wenig wie möglich und so unverfänglich wie nötig über» die Vergangenheit « sprach.

Von Weizsäcker spielte seit der Göttinger Erklärung eine wachsende öffentliche Rolle. Als Physiker und Philosoph wurde er quasi zum vereinten Humboldt (Alexander samt Wilhelm), einer Instanz, die das Land mit Einsichten über nuklearen Krieg und Frieden, die wissenschaftlich-technische Welt, Quantenphysik, Weltinnenpolitik und das Religiöse bediente. Mit allem, was den jungen von Weizsäcker bewegt hatte, konnte der ältere von Weizsäcker nichts mehr anfangen. Diskussionen darüber konnten seine nationale Sonderstellung bloß gefährden. Daher ist es nicht verwunderlich, dass er sich ungern zu seinem früheren Selbst äußerte und nur unter Druck historische Eingeständnisse machte. Aussagen ohne Wenn und Aber sind folglich selten und stammen aus einer sehr späten Phase: "Ich habe falsch gehandelt $\varkappa^{8}$ ist ein Beispiel. Dieser Satz enthält ein generelles Schuldbekenntnis; eine Offenlegung seiner Entwicklungsgeschichte als Atomphysiker im Dritten Reich enthält er nicht. Als dann in den späten 1980er Jahren die unfriedlichen Konturen der Lebensphase von 1939 bis 1945 durch grundlegende historische Arbeiten wie die von Mark Walker deutlicher wurden und in den Medien zunehmend auf Interesse stießen, belobigte von Weizsäcker den jungen Wissenschaftshistoriker zwar für sei-

5 Hoffmann 1993 b, S. 172 f.

6 Damit sind Werke gemeint wie Kriegsfolgen und Kriegsverbütung (Weizsäcker et al. 1971); Der bedrohte Friede (Weizsäcker 1981); Bewußtseinswandel (Weizsäcker 1988).

7 Für von Weizsäckers innere Abrechnung mit sich selbst sprechen beispielsweise folgende Verse, die er nach eigener Angabe im August 1945 niederschrieb: »Ich ließ mit sehendem Aug in dunklen Jahren / schweigend geschehn Verbrechen um Verbrechen. / Furchtbare Klugheit, die mir riet Geduld! / Der Zukunft durft ich meine Kraft bewahren, / allein um welchen Preis! Das Herz will brechen. / O Zwang, Verstrickung, Säumnis! Schuld, o Schuld! «; Weizsäcker 1981, S. 572 f.

8 Weizsäcker 1991 b, S. 232. 
nen Fleiß, aber erklärte ihn auch als dem Verständnisproblem nicht recht gewachsen. ${ }^{9}$

Mit anderen Worten: Der Lernprozess von Weizsäckers kam in der frühen Nachkriegszeit zu einem Abschluss in der Göttinger Erklärung, welche einen öffentlichen Lernprozess auslöste und einen Neuanfang mit sich brachte. Dieser Lernprozess war der Lernprozess der deutschen Gesellschaft über das komplexe Bündel der »Atomfragen «. Die nationale Atom-Diskussion war hauptsächlich vorwärtsschauend, was in der Natur der Sache lag und von Weizsäckers Intentionen entgegenkam, der allein darüber reden wollte, »was wir künftig tun wollen «. Dass es eine militärisch ausgerichtete Vorgeschichte im Dritten Reich gab, wurde nicht offen thematisiert. Einzelheiten waren weitgehend unbekannt und blieben im Interesse des deutschen Neuanfangs ausgeblendet. Die Rekonstruktion dieser Vorgeschichte hat sich über Jahrzehnte hingezogen und ist noch immer nicht abgeschlossen. Von Weizsäcker, Heisenberg und andere, wie von Weizsäckers Freund Karl Wirtz, ${ }^{10}$ unterzeichneten die Göttinger Erklärung im öffentlichen Bewusstsein nicht als die ersten Atomphysiker Hitlerdeutschlands, sondern als führende Wissenschaftler Westdeutschlands. ${ }^{11}$

Der gesellschaftliche Lernprozess involviert eine Vielzahl von Menschen, ist aber als solcher nicht grundverschieden vom individuellen Lernprozess. Letzteren hat von Weizsäcker zwar nie mit konkretem Bezug auf seinen Beitrag zum deutschen Atomwaffenprojekt beschrieben, aber im Gefolge seines Onkels Viktor von Weizsäcker ${ }^{12}$ an mehreren Stellen methodologisch als »Kreisgang " gedeutet. Von Weizsäcker wandte den »scheinbaren Zirkel «, in dem objektive und subjektive Bezüge ineinandergreifen und sich gegenseitig erhellen, im Sommersemester 1946 in einer ersten Vorlesung nach dem Krieg als Honorarprofessor in Göttingen auf die Natur im Verein mit den Natur- und Geisteswissenschaften an. Natur und Wissenschaft bilden demnach zwei komplementäre Halbkreise, die einen Vollkreis ergeben, der

9 Siehe Walker 1989 und den Brief von Weizsäckers an Walker vom 5. August 1990, worin es heißt: "So etwas kann man nur sagen, wenn man nicht dabeigewesen ist. Das heißt, ich bewundere Ihre Anstrengung, zu verstehen, ich bewundere aber nicht ganz im selben Grade den Erfolg dieser Anstrengung «; Weizsäcker, Hora 2002, S. 277.

10 Wirtz war Mitbegründer des Kernforschungszentrums Karlsruhe und verantwortlich für den ersten Kernreaktor in Deutschland (Forschungsreaktor 2: Baubeginn 1957, Inbetriebnahme 1962, Abschaltung 1981, Stilllegung 1996). In seinen Erinnerungen betonte Wirtz, dass sich dieser Reaktor »in seinem Grundkonzept an die schon im Jahre 1940 von Heisenberg in zwei Arbeiten ausgeführten Grundzüge der Theorie der Kernreaktoren anschloß «; Wirtz 1988, S. 98.

1116 von den 18 Unterzeichnern hatten »einst im >Uranverein` mitgearbeitet « (Müller 1990, S. 553). Die Ausnahmen sind Friedrich Paneth, der es vorgezogen hatte, 1933 von einer englischen Vortragsreise nicht nach Deutschland zurückzukehren, und Heinz Maier-Leibnitz, der während des Krieges zwar bei Walther Bothe in Heidelberg, aber nicht für den Uranverein gearbeitet hatte; siehe ebd., S. 632, Anmerkung 127.

12 Weizsäcker 1973 [1940].

Leviathan, 41. Jg., 3/2013 
mehrmals zu durchlaufen ist. ${ }^{13}$ Für von Weizsäckers Lernprozess gilt ähnliches: Seine begeisterte Mitarbeit am Atomwaffenprogram des Dritten Reichs, die älter als sein Nachdenken über diese Kooperation war, wurde zum Ausgangspunkt seines persönlichen Lernprozesses. Gleichwohl trifft aber auch zu, dass von Weizsäcker erst im Gefolge seiner späteren Reflexionen in die Lage versetzt wurde, voll zu verstehen, woran er zunächst einmal de facto teilgenommen hatte.

Das, was für von Weizsäcker individuell begreiflich wurde, ist lange Zeit gesellschaftlich unbegreiflich geblieben. Ein Grund dafür war, dass von Weizsäcker beschlossen hatte, der Welt nur die Ergebnisse seiner Reflexionen mitzuteilen, nicht jedoch die Einzelheiten der realen Geschichte, die er im Gehäuse seines privaten Nachdenkens unter Ausschluss der Öffentlichkeit verarbeitete. Das ist der »unvollendete Kreisgang «, den wir jetzt für den gesellschaftlichen Lernprozess mit Rekurs auf die nukleare Geschichte Deutschlands von 1939 bis 1945 nachholen ganz im Sinne des von Weizsäcker so geschätzten epistemologischen »Zirkels der Erkenntnis «.

\section{Wendepunkt 1945: Die »friedliche Entwicklung « der Atomenergie}

"Die Geschichte wird festhalten, daß die Amerikaner und die Engländer eine Bombe bauten und daß zur selben Zeit die Deutschen unter dem Hitler-Regime eine funktionsfähige Maschine herstellten. Mit anderen Worten, die friedliche Entwicklung der Uranmaschine fand in Deutschland unter dem Hitler-Regime statt, während die Amerikaner und die Engländer diese gräßliche Kriegswaffe entwickelten. «14

Carl Friedrich von Weizsäcker, 7. August 1945

Am Tage nach der Zündung der ersten Atombombe artikulierte der 33-jährige von Weizsäcker den nuklearen Phasensprung des »Uranvereins « (der mit Werner Heisenberg verbundenen Sparte des deutschen Atomwaffenprogramms) von der militärischen zur pazifistischen Anwendung der Atomenergie. Der Ort, an dem dieser Umschwung seinen Anfang nahm, war »Farm Hall«, ein englisches Landhaus in der Nähe von Cambridge, in dem von Weizsäcker und neun weitere deutsche Atomwissenschaftler nach ihrer Gefangennahme im April und Mai 1945 für genau ein

13 Weizsäcker 1954 [1948], S. 8: Im ersten Durchgang wird argumentiert, dass - da die Natur älter als der Mensch und der Mensch ein Naturwesen ist - die Naturwissenschaft eine Voraussetzung der Geisteswissenschaft ist. Im zweiten Durchgang wird davon ausgegangen, dass - da die Menschen älter als die Naturwissenschaft sind und Naturwissenschaft von Menschen gemacht wird - Geisteswissenschaft eine Voraussetzung von Naturwissenschaft ist.

14 Hoffmann 1993 b, S. 172 f. 
halbes Jahr (vom 3. Juli 1945 bis 3. Januar 1946) interniert waren. ${ }^{15}$ Der englische Geheimdienst hatte Farm Hall mit verborgenen Mikrofonen ausgestattet. Die Gespräche der unfreiwilligen guests of His Majesty wurden aufgezeichnet, abgehört und übersetzt. ${ }^{16}$ Die aufschlussreichen Gespräche der zehn Internierten ${ }^{17}$ deuten an, dass sie nicht übermäßig besorgt über die Möglichkeit permanenter Bespitzelung waren. Der Weg ums entlegene Rosenbeet im Garten »für ernste Gespräche zu zweit « 18 war also möglicherweise mehr dazu angetan, sich von den Ohren der deutschen Kollegen zu entfernen als von denen der englischen Bewacher. Die Existenz geheimer Abhörprotokolle wurde bereits 1962 bekannt, ${ }^{19}$ ihre Veröffentlichung jedoch vom britischen Oberhaus erst 1992 genehmigt. ${ }^{20} 1981$ hatte von Weizsäcker in einem Brief an seinen Freund Edward Teller erklärt, dass er es am besten fände, »wenn die Bänder noch ein paar Jahrzehnte « unveröffentlicht blieben, da die jüngeren Historiker unfähig seien, »sich in den Äußerungsstil und die Denkformen früherer Generationen einzufühlen «. ${ }^{21}$

Die Verlagerung der militärischen Urgeschichte auf die angelsächsische Seite war einleuchtend, denn von Weizsäckers »Sprachregelung « 22 zum deutschen Atomprojekt, »daß wir gar nicht wollten, daß die Sache gelingt ", ${ }^{23}$ schien den in Farm Hall Internierten aus der Klemme zu helfen. Von Weizsäckers kühne Formulierung versprach, den Vorwurf der Inkompetenz (wenn auch nicht des »Volksverrats «) abzuwehren und zugleich eine Brücke zwischen dem Gestern und dem Heute zu ermöglichen sowie das Schuldempfinden einiger (Otto Hahn vor allem) zu dämpfen.

15 Ein englisches Gesetz erlaubte es, im Kriege Personen des Feindes, die nicht Soldaten waren, für die Dauer von bis zu sechs Monaten ohne Begründung festzuhalten (detain). Die deutschen Hausgäste von George VI. bezeichneten sich daher als die »Detainten «; siehe Hoffmann 1993 a, S. 26 f., 49.

16 Die Gespräche wurden von sechs bis acht Maschinen auf metallenen Schellack-Disketten aufgezeichnet (nicht tapes, Bänder oder Tonbänder), die regelmäßig neu beschichtet und eingesetzt wurden. Es wird angenommen, dass sich keine Disketten mit Original-Aufnahmen erhalten haben. Die deutschen Transkriptionen sind, wie es scheint, ebenfalls nicht mehr vorhanden. Ungefähr zehn Prozent des aufgenommenen Tonmaterials sollen vom Deutschen ins Englische übersetzt worden sein; siehe Frank 1993, S. 12. Die deutsche Veröffentlichung der Protokolle ist eine Rückübersetzung aus dem Englischen.

17 Das waren (in alphabetischer Reihenfolge): Erich Bagge (1912-1996), Kurt Diebner (1905-1964), Walther Gerlach (1889-1979), Otto Hahn (1879-1968), Paul Harteck (1902-1985), Werner Heisenberg (1901-1976), Horst Korsching (1912-1998), Max von Laue (1879-1960), Carl Friedrich von Weizsäcker (1912-2007) und Karl Wirtz (1910-1994).

18 Heisenberg 1969, S. 264.

19 Leslie Groves, der militärische Leiter des Manhattan Projekts, zitierte damals ausgewählte Passagen erstmals ausführlich in seinen Memoiren; siehe Groves 1962, S. 333-340. Siehe auch SPIEGEL 1962.

20 Hoffmann 1993 a, S. 51.

21 Weizsäcker, Hora 2002, S. 144, 142.

22 Hoffmann 1993 a, S. 43.

23 Hoffmann 1993 b, S. 155. 
Darüber hinaus gab sie den deutschen Wissenschaftlern den Nimbus der moralischen Überlegenheit im Vergleich mit den angelsächsischen und ihrer "gräßlichen Kriegswaffe«. Von Weizsäckers Interpretationspolitik überzeugte daher die Mehrzahl der Internierten. Der Nachteil dieser Manipulation der Geschichte war freilich der Zwang, die Vergangenheit des deutschen Projekts zu verfälschen; denn die Parole hieß ja nicht: Von nun an werden wir nur noch friedliche Kernforschung betreiben, sondern: Das haben wir doch von Anfang an getan. Dieser Nachteil fiel 1945 nicht sonderlich ins Gewicht, hat sich aber später gegen von Weizsäcker selbst ausgewirkt, da er von allen Beteiligten am längsten lebte und daher immer öfter gezwungen war, wichtige Auslassungen zuzugeben und kontrafaktische Erinnerungen » unablässig « zu erklären.

Die Verteidigungsstrategie der Atomwissenschaftler des Dritten Reichs war natürlich nur den Internierten, ihren verborgenen Zuhörern und dem kleinen Kreis der Rezipienten der Protokolle bekannt. Die erste (missverstandene) Andeutung, dass es Ohrenzeugen gegeben haben konnte, kam jedoch bald. ${ }^{24}$ Samuel Goudsmit, der wissenschaftliche Leiter der amerikanischen Spezialeinheit, die die zehn deutschen Atomwissenschaftler im Frühjahr 1945 gefangen genommen hatte, brachte 1947 ein Buch über seine »hush-hush Mission « unter dem Titel Alsos heraus, dem Tarnnamen seines Kommandounternehmens. ${ }^{25}$ Darin berichtete er über die »brillante Rationalisierung " des deutschen "Versagens ${ }^{26}$ und » die neue Erkennungsmelodie « (the new theme song) der deutschen Wissenschaftler: "Germany worked on the uranium problem for peaceful uses only; the Allies, for purposes of destruction ${ }^{27}{ }^{27} \mathrm{Im}$ Übrigen betonte Goudsmit wiederholt die vermeintliche Unkenntnis der deutschen Physiker über die Konstruktion einer Atombombe und den Kernsprengstoff Plutonium. Für die von Weizsäcker'sche Sprachregelung war das der erste unfreundliche Test.

Wenn man sich fragt, was wiegt schwerer für einen von Weizsäcker mit seinem damaligen " maßlosen Ehrgeiz und Hochmut ${ }^{2}{ }^{28}$ beziehungsweise einen Heisenberg, der es weder in der Physik noch im Schachspiel oder Skilaufen ertragen konnte, Zweitbester zu sein: der Vorwurf, an einer deutschen Atombombe gearbeitet zu haben, oder die Bemerkung, »he and his colleagues had completely missed the basic principle of the atom bomb ${ }^{29}$ dann kann man die Zwangslage verstehen, in der

24 Dass dieses Mithören vor allem auf Mikrofonen beruhte, wurde zunächst nicht realisiert. Von Weizsäcker identifizierte beispielsweise nur die beiden englischen Offiziere, die den Internierten beigegeben waren, als mögliche Berichterstatter; siehe Jungk 1964, S. 203.

25 Buchumschlag: »The exciting and sometimes incredible story of a strange Mission of scientific sleuths in pursuit of the German atom bomb. The startling findings of this hushhush Mission [...] increase daily in vital importance for all of us «.

26 Goudsmit 1947, S. 138: »[...] some of the younger men hit upon a brilliant rationalization of their failure. They would turn that very failure to their advantage by denying that they had ever tried to make an atomic explosive ".

27 Ebd., S. 139.

28 Weizsäcker 1977, S. 556.

29 Goudsmit 1947, S. 138. 
sich von Weizsäcker und Heisenberg in der Auseinandersetzung mit Goudsmit befanden. Die wissenschaftliche Ehre verlangte es, darauf zu bestehen, dass man die Möglichkeit der Produktion von Plutonium in einer Uranmaschine erkannt hatte, während die politische Strategie es ratsam erscheinen ließ, die Verweigerung einer deutschen Superbombe seitens der deutschen Wissenschaftler zu behaupten. In ihrer Antwort auf diese Herausforderung haben Heisenberg und von Weizsäcker sowohl ihre tatsächliche Sachkenntnis als auch ihre vorgebliche Zurückhaltung verteidigt. $^{30}$

Der Eindruck, dass die deutschen Fachleute eine hinhaltende Taktik gegenüber dem deutschen Streben nach Atomwaffen eingenommen hatten, den Heisenberg 1946 in Umlauf brachte ${ }^{31}$ und Goudsmit 1947 mit Alsos attackierte, wurde 1956 verstärkt, als Robert Jungk seinen Bestseller Heller als tausend Sonnen veröffentlichte. Die Physiker des Zweiten Weltkriegs wurden darin als tragische Heroen und bahnbrechende Wissenschaftler geschildert, die »zwischen Forscherdrang und Gewissensqual « beziehungsweise »Erkenntnislust und Gewissensnot « ${ }^{32}$ schwankten, wobei Jungk Drang und Lust den angelsächsischen, Qual und Not den deutschen Physikern zuschrieb:

»Es erscheint paradox, daß die in einer säbelrasselnden Diktatur lebenden deutschen Kernphysiker, der Stimme ihres Gewissens folgend, den Bau von Atombomben verhindern wollten, während ihre Berufskollegen in den Demokratien, die keinen Zwang zu befürchten hatten, mit ganz wenigen Ausnahmen sich mit aller Energie für die neue Waffe einsetzten $« .33$

Im »Dank des Verfassers « erwähnte Jungk, dass von Weizsäcker ihm unabgeschlossene »Bemerkungen zur Atombombe « von 1945 und unveröffentlichte Notizen zu Goudsmits Alsos gab, ${ }^{34}$ und in seinen Lebenserinnerungen, dass von Weizsäcker ihn zum Privatgespräch »im Arbeitszimmer seiner Göttinger Wohnung « empfing. ${ }^{35}$ Dass der jüdische Emigrant Jungk die deutschen Physiker als sachkundig beschrieb und im Vergleich zu den amerikanischen mit kritischem Misstrauen ihrem Staat gegenüber auszeichnete, empfanden von Weizsäcker und Heisenberg als verdiente

30 Walker 1990 b. Heisenbergs Richtigstellungen von 1948/49 reichen von der Schwäbischen Donau-Zeitung bis zur New York Times; siehe Blum et al. 1989, S. 33-42.

31 Heisenberg 1946, S. 32: »Die Fachleute haben nicht versucht [...] bei der höchsten Führung einen großen Industrieeinsatz für die Herstellung von Atombomben zu erwirken. Die deutschen Physiker hatten von vornherein darauf hingearbeitet, die Kontrolle über das Vorhaben in der Hand zu behalten, und sie haben den Einfluß, den sie als Sachverständige hatten, darauf verwendet, die Arbeiten in dem in diesem Bericht geschilderten Sinne zu lenken. Die äußeren Umstände haben ihnen die schwere Entscheidung, ob sie Atombomben herstellen sollten, aus der Hand genommen «.

32 Jungk 1956. Das erste Zitat ist dem Vorsatzblatt der Rowohlt Taschenbuchausgabe (1964) (»Zu diesem Buch«) und das zweite dem hinteren Buchdeckel dieser Ausgabe entnommen.

33 Jungk 1956, S. 112; Jungk 1964, S. 102.

34 Jungk 1956, S. 8.

35 Jungk 1993, S. 298.

Leviathan, 41. Jg., 3/2013 
publizistische Ehrenrettung gegenüber Goudsmit. ${ }^{36}$ In der dritten Auflage von $\mathrm{Hel}$ ler als tausend Sonnen, die nach meinen Recherchen 1958 erschienen sein muss, hatte Jungk sein Nachwort im Lichte der Göttinger Erklärung erweitert. Das veränderte Nachwort gipfelte nun darin, dass sich die deutschen Physiker mit der »feierlichen Verpflichtung " gegen den »Mißbrauch ihrer Wissenschaft « weltweit beispielhaft verhalten hätten und »ihrer bereits in den Jahren des >Dritten Reichs größten Gefahren praktizierten Widerstandshaltung weiterhin treu « geblieben seien. ${ }^{37}$ Von deutscher Seite wurde dieser Heroisierung anfänglich nicht widersprochen.

In einem außerordentlich freundlichen Brief an Jungk vom Januar 1957 wandte sich von Weizsäcker nicht gegen die Hochstilisierung der deutschen Physiker zu Gewissenshelden, sondern gegen Jungks Kritik an Teller und Robert Oppenheimer. So beklagte von Weizsäcker beispielsweise, dass Jungk den Bericht über seine frühen Gespräche mit Teller »zuungunsten von Teller « ausgelegt habe. ${ }^{38}$ Oppenheimer gestand er eine »verhältnismäßig hohe Begabung « zur kritischen Selbstanalyse zu, gepaart mit der Weigerung, »die Ergebnisse einer solchen Analyse der Mitwelt mitzuteilen « ${ }^{39}$ Wenn hinter von Weizsäckers Verteidigung der amerikanischen Physiker die vorausschauende Einsicht stand, dass die Jungk'sche Kritik in dem Moment, in dem die "Widerstandshaltung « der deutschen Physiker als Fiktion erkannt werden würde, auch ihn treffen müsste, dann begann die Selbstverteidigung von Weizsäckers 1957 mit der Verteidigung der amerikanischen Vaterfiguren der Atom- und Wasserstoffbombe. ${ }^{40}$

Zweifel am Widerstand der deutschen Physiker kamen außerhalb Deutschlands schnell auf. Niels Bohr war »greatly amazed «, ${ }^{41}$ als er 1957 die dänische Ausgabe von Jungks Buch sah, die im Anhang erstmals auch einen Briefauszug Heisenbergs

36 Weizsäcker, Hora 2002, S. 285: »Wenn man denkt, daß wir vorher unter den vollkommen absurden Anwürfen von Goudsmit standen, war man eben dankbar, wenn einmal jemand kam, der sah, daß wir in Wirklichkeit die schlimmen Motive, die Goudsmit vorausgesetzt hatte, nicht gehabt hatten «.

37 Jungk 1964, S. 309. Das Nachwort in der 1. und 2. Auflage (1956 und 1957) ist unverändert. Der Hinweis auf die Göttinger Erklärung kann also erst in der 3. Auflage erfolgt sein. Trotz intensiver Suche in den Bibliotheken im deutschsprachigen Raum habe ich aber nur eine Sonderausgabe des »Europäischen Buchklubs « gefunden (ohne Erscheinungsjahr und Auflagenangabe), die eine Lizenzausgabe des Scherz \& Goverts Verlags war und seinerzeit mit dem Erscheinungsjahr 1958 inventarisiert wurde. Diese Buchklubausgabe enthält Jungks Lob der Göttinger Erklärung in einem »Epilog ", der dem Nachwort der späteren Ausgaben entspricht. Ich nehme daher an, dass die 3. Auflage mit dem veränderten Nachwort erstmals 1958 erschien.

38 Weizsäcker, Hora 2002, S. 49.

39 Ebd., S. 51.

40 Siehe ebd., S. 52 f.: "So könnte es doch sein, daß die Sicherung des Weltfriedens, die vielleicht durch die Wasserstoffbombe im Augenblick erreicht ist, eine angemessene Belohnung Tellers für den inneren moralischen Einsatz ist, den er hier geleistet hat «.

41 Bohr 2002. 
an den Verfasser (Jungk) enthielt. ${ }^{42}$ Bohr war der Besuch Heisenbergs und von Weizsäckers im September $1941 \mathrm{im}$ besetzten Kopenhagen nicht als ein » Friedensfühler « der »Passivisten« unter den deutschen Atomforschern « in Erinnerung geblieben. ${ }^{43}$ Er erinnerte sich, dass Heisenberg und von Weizsäcker ihre feste Überzeugung bekundeten, dass Deutschland den Krieg gewinnen würde und deshalb Kooperation mit den Deutschen angeraten sei. Heisenberg hatte bei Bohr den Eindruck hinterlassen, »everything was being done in Germany to develop atomic weapons « $\cdot{ }^{44}$ Die von Jungk kolportierte Erklärung, der Besuch habe »die Weitergabe des 'negativen Geheimnisses« « bezweckt, »daß die Deutschen keine Atombombe zu bauen beabsichtigten " und "die Herstellung dieser aus moralischen Gründen verwerflichen Waffe durch eine stillschweigende Übereinkunft der deutschen und alliierten Atomforscher verhindern « 45 wollten, war für Bohr unfassbar. ${ }^{46}$ Ungläubig und verblüfft war auch die Reaktion in den Vereinigten Staaten, als dort 1958 die amerikanische Übersetzung von Jungks Buch erschien. Hans Bethe, der maßgeblich am Atombombenprogramm der USA beteiligt gewesen war und sowohl alle Beteiligten als auch die wissenschaftliche Materie kannte, stellte unverzüglich die Behauptung infrage, dass moralische Gründe Heisenberg und von Weizsäcker vom Bau einer Atombombe abgehalten hätten. ${ }^{47}$

Jungk hatte Schützenhilfe geleistet und das Ansehen der deutschen Physiker im Inland gefestigt, aber Heisenberg und von Weizsäcker konnten weder Bohr noch Bethe einfach widersprechen. Sie relativierten daher Jungks Darstellung und betonten, dass sie es eigentlich den "äußeren Umständen « verdankten, nicht in die Ver-

42 Zwei Briefe Heisenbergs an Jungk sind bekannt: ein erster vom 17. November 1956, der sich auf die Erstausgabe von Heller als Tausend Sonnen bezieht, und ein zweiter vom 18. Januar 1957, der Heisenbergs Version des Gesprächs mit Bohr enthält (das Thema des weltbekannten Theaterstücks Copenhagen; siehe Frayn 2000; Dörries 2005). Im ersten Brief greift Heisenberg die Legende von der friedlichen und unfriedlichen Atomforschung auf, die von Weizsäcker vor- und weitergegeben und Jungk mit der Behauptung eines aktiven Widerstands verbunden hatte. Im Anschluss an die kategorische Feststellung, dass in einer Diktatur » aktiver Widerstand nur von Leuten ausgeübt werden [kann], die scheinbar beim System mitmachen ", erklärte Heisenberg, dass er selbst keinen Widerstand gegen Hitler geleistet habe, aber wiederum auch, »daß wirklicher Widerstand nur von Leuten kommen kann, die scheinbar mitspielen « (Heisenberg 1956/57; Hervorhebung W.S.). Aus Verlautbarungen dieser Art hat Jungk die Fiktion des passiven Widerstands der deutschen Physiker abgeleitet.

43 Jungk 1956, S. 109, 108; Jungk 1964, S. 99, 98.

44 Siehe www.nba.nbi.dk/papers/docs/d01tra.htm (Zugriff vom 19. Mai 2013).

45 Jungk 1956, S. 109; Jungk 1964, S. 99.

46 Dass Heisenberg und von Weizsäcker ihrem hochverehrten Mentor »helfen « wollten, hat Bohr nie bezweifelt. Dass sie ihn dazu bewegen wollten, die Ablehnung jeglicher Kollaboration im besetzten Dänemark aufzugeben, um es ihnen zu ermöglichen, für Bohr persönlich und sein Institut fachlich einzutreten, ist eine plausible Hypothese; siehe Schwartz 2011, S. $434 \mathrm{f}$.

47 Bethe 1958.

Leviathan, 41. Jg., 3/2013 
suchung geraten zu sein, Atombomben zu bauen. ${ }^{48}$ Für Jungk glich das freilich einem Denkmalssturz. Auf einmal sollte das, was er von seinen beiden Informanten gehört und gutgläubig aufgeschrieben hatte, als Jungk'sche Überhöhung verstanden werden. Von Weizsäcker verwandelte den »Lieben Herrn Jungk «, dessen Brief ihm noch 1957 »eine ganz große Freude" gewesen war, zunächst in eine anima candida und schließlich zum »Fanatiker «. ${ }^{49}$ Jungk hingegen fühlte sich von »dem einst verehrten Mann « 50 verraten und schrieb:

»Von den zahlreichen Atomforschern, die ich in den Jahren 1953 bis 1955 in Europa und Amerika interviewte, hat keiner mich so stark beeindruckt wie Leo Szilard, keiner so enttäuscht wie Robert Oppenheimer und keiner - vielleicht unabsichtlich - mich so irregeführt wie Carl Friedrich von Weizsäcker «. ${ }^{51}$

Was die von Weizsäcker angerufene Autorität der Geschichte »festgehalten « hat, ist, dass die »friedliche " deutsche Atomgeschichte während des Zweiten Weltkriegs eine bewusst irreführende »Lesart « ${ }^{52}$ war. Von Weizsäckers Versuch, den Blick zurück zu manipulieren, um den Blick nach vorn (und die Karriere) in eine von der Vergangenheit unbeschwerte Zukunft freizugeben, hat historische Spuren sowohl verwischt als auch hinterlassen, beispielsweise im Gefolge der Wirkungsgeschichte Jungks. Als 1992 in Die Zeit von dem Journalisten Hermann Jensen der Gedanke geäußert wurde, »die Gefahr, daß eine deutsche Atombombe gebaut wurde, war viel größer, als man bisher angenommen hat « ${ }^{53}$ konterte der letzte Doktorand und

48 SPIEGEL 1957. Siehe Nr. 19 (S. 46) für Heisenberg und Nr. 22 (S. 48) für von Weizsäcker.

49 Weizsäcker, Hora 2002, S. 251. Nach von Weizsäcker ist die Widerstandsgeschichte Jungks Problem: »Ich habe immer gesagt, daß dieses [die Absprache oder Verschwörung, die Bombe nicht zu bauen] eine übertriebene Vorstellung ist, die Jungk damals entwickelt hat. Und Jungk hat sie ja auch inzwischen aufgegeben «; siehe Dönhoff, Sommer 1992, S. 10. Von Weizsäcker bezieht sich hier auf Jungks Erklärung in der Einleitung zu Walker 1990 a, S. 7: »Daß ich [...] dazu beigetragen habe, den Mythos vom passiven Widerstand der bedeutendsten deutschen Physiker zu verbreiten, ist vor allem auf meine Hochschätzung dieser eindrucksvollen Persönlichkeiten zurückzuführen, die ich seither als unangebracht erkennen mußte «.

50 Jungk 1993, S. 299: »Um sich von dem Vorwurf zu befreien, er habe gemeinsam mit Heisenberg eine Legende in die Welt gesetzt und mich zu ihrer Verbreitung benutzt, behauptete Weizsäcker zuerst nur, ich sei >naiv gewesen, dann aber, als ich mich dagegen leider nicht sofort wehrte, die ganze Geschichte vom Passivismus deutscher Atomphysiker sei eigentlich nur meine >Idee< gewesen, obwohl er doch nachweislich diese Behauptung schon Jahre, bevor er mich traf, vor Zeitzeugen verbreitet hatte .

51 Ebd., S. 285 f.

52 Diese treffende Bezeichnung der von Weizsäcker'schen Sprachregelung stammt von Laue, der den Ausdruck 1959 in einem Brief an Paul Rosbaud gebrauchte: "Allmählich entwickelte sich dann auch, in Tisch-Gesprächen, die Lesart, die deutschen Kernphysiker hatten die Atombombe gar nicht haben wollen, sei es, weil sie es während der zu erwartenden Kriegsdauer für unmöglich hielten, sei es, weil sie überhaupt nicht wollten. Führend bei diesen Diskussionen war Weizsäcker. Ethische Gesichtspunkte habe ich dabei nicht gehört. Heisenberg saß zumeist stumm dabei «; siehe Bernstein 2001 [1996], S. 351.

53 Jensen 1992, S. 41. 
Mitherausgeber der Werke Heisenbergs, Helmut Rechenberg, »die moralische Debatte um Atombomben [sollte man] in erster Line auf die wirklich betroffenen alliierten Kollegen beschränken «. .54

\section{Gruppendynamik: Heisenbergs »Uranverein « gegen Diebners "Forschungsgruppe«}

»Ich ging zu Diebner und sagte ihm, es wäre doch für unsere Arbeiten ausgezeichnet, wenn wir Heisenberg [...] immer zur Verfügung hätten, und der gute Diebner ist darauf reingefallen und hat zugestimmt. «55

Carl Friedrich von Weizsäcker, 3. Juni 1993

Der Uranverein stand lange Zeit im Fokus der nuklearen Wissenschaftsgeschichte des Dritten Reichs. Dafür sorgte schon die Prominenz seiner führenden Vertreter, Heisenberg und von Weizsäcker, aber auch das in Deutschland über Jahrzehnte wachsende und vor allem von der Wochenzeitschrift Der Spiegel immer neu genährte Gefühl, dass die story der deutschen Hauptfiguren des Uranvereins lückenhaft und unstimmig war. Wenn man die Namensliste der zehn Farm-Hall-Internierten mit der der Göttinger 18 vergleicht, fällt auf, dass es sechs Überschneidungen und vier Ausnahmen gibt: Bagge, Diebner, Harteck und Korsching gehörten zur Farm-Hall-Gruppe, waren aber nicht an der Göttinger Erklärung beteiligt. Für die beiden Erstgenannten ist der Hauptgrund klar: Sie waren Mitglieder der NSDAP $^{56}$ und daher nicht geeignet, den von Weizsäcker und Heisenberg orchestrierten Neuanfang der Physik in Deutschland unbelastet zu vertreten. ${ }^{57}$ Gegen Harteck und Korsching sprach, dass sie Experimentalphysiker waren und deshalb in den Augen der tonangebenden Theoretiker nicht zu den wirklich »brillanten Physikern « gehörten. ${ }^{58}$ Harteck, der seine Professur für physikalische Chemie in

54 Rechenberg 1992.

55 Weizsäcker 1993, S. 342 f.

56 Nach Auskunft des Bundesarchivs in Berlin (ehemals BDC) hatte Diebner die NSDAPMitgliedsnummer 1834823 (Eintrittsdatum 01.04.1933) und Bagge 3664921 (Eintrittsdatum 01.05.1935). Bagge sowie Harteck waren außerdem Mitglieder des Nationalsozialistischen Lehrerbundes.

57 Heisenberg im Gespräch mit Patrick Blackett im September 1945: »Die einzigen aus unserer Gruppe, die in der NSDAP waren, sind Diebner und Bagge " (Hoffmann 1993 b, S. 238). Für Diebners Erklärung dieses Umstands siehe ebd., S. 125; für Bagges siehe ebd., S. $123 \mathrm{f}$.

58 Heisenberg zu Blackett; siehe Hoffmann 1993 b, S. 236: »Er [Korsching] ist kein brillanter Physiker, dafür aber ein sehr guter Experimentator; was die Isotopentrennung betrifft, hatte er eine hübsche Idee«. 
Hamburg wiederaufnehmen konnte und dort auch 1948-1950 Rektor wurde, war 1951 in die USA gegangen. ${ }^{59}$

Die Vorrangstellung der akademischen Elite im Allgemeinen und der theoretischen Physiker im Besonderen war - aller nationalsozialistischen Revolutionsrhetorik zum Trotz - ein Traditionsproblem des deutschen Uranprojekts. Die Theoretiker standen an der Spitze, und die Experimentatoren spielten eine zweitrangige Rolle. Nach den BBC-Nachrichten über Hiroshima am Abend des 6. August 1945 und der plötzlichen Erkenntnis, dass das deutsche Projekt vergleichsweise weit zurückgeblieben war, kam dieses Problem zur Sprache. Zum großen Missfallen Walther Gerlachs, der zwar wie Diebner, Bagge, Harteck, Korsching und Wirtz auch »nur « Experimentalphysiker, aber durch Alter und Stellung herausgehoben war, ${ }^{60}$ erklärte Korsching: »Das beweist jedenfalls, daß die Amerikaner zu wirklicher Zusammenarbeit in ungeheurem Ausmaß fähig sind. In Deutschland wäre das unmöglich gewesen. Jeder behauptete, der andere sei inkompetent « ${ }^{61}$ Gerlach, der für das Gelingen des Uranprojekts verantwortlich zeichnete, lobte daraufhin die enge Zusammenarbeit und das große Vertrauen im deutschen Projekt. Korschings Einwand, die Inkompetenz sei natürlich » nicht offiziell « behauptet worden, beantwortete Gerlach »brüllend «: »Auch nicht inoffiziell. Widersprechen Sie mir nicht «. ${ }^{62}$ Später setzte Korsching noch hinzu: »Die Amerikaner konnten es eben besser als wir, das ist mal klar «. Das Abhörprotokoll vermerkte daraufhin » Gerlach verläßt den Raum «. 63

Harteck, der das Heereswaffenamt (HWA) seit 1937 über chemische Waffen beriet, das Reichskriegsministerium im Frühjahr 1939 auf die militärische Relevanz der Kernspaltung hinwies und mit seinem ganzen Institut in Hamburg an der Isotopentrennung sowie der Neutronenmoderation durch Schweres Wasser gearbeitet hatte, brachte die Frustration der "Zweitrangigen « auf den Punkt:

»Natürlich haben wir die Sache eigentlich nicht richtig angefangen. Die Theorie wurde als das Wichtigste betrachtet und die Experimente als Nebensache, und dann wurden fast

59 Harteck lehrte 1951-1968 am Rensselaer Polytechnic Institute; siehe Hentschel 1996, S. XXXI. Hentschels Appendix F (Biographical Profiles, S. XVIII-LIII) ist ausgezeichnet für erste Angaben über personen- und karrierebezogene Daten.

60 Als Senator der Kaiser-Wilhelm-Gesellschaft (KWG) und Leiter der Arbeitsgemeinschaft für Kernphysik im Reichsforschungsrat (RFR) sowie seit Januar 1944 Hermann Görings »Bevollmächtigter für kernphysikalische Forschung « war Gerlach für Heisenberg und von Weizsäcker respektabel und von unumgänglicher Bedeutung; siehe Hentschel 1996, S. XXVII. Auch in der Bundesrepublik nahm Gerlach hervorragende Positionen ein: Rektor der Münchner Universität (1948-51), Präsident der Fraunhofer-Gesellschaft (1949-51), Präsident der Deutschen Physikalischen Gesellschaft (1956-57) und Vizepräsident der Deutschen Forschungsgemeinschaft (1951-61).

61 Hoffmann 1993 b, S. 152.

62 Ebd.

63 Ebd., S. 154. Als Gerlach nach dem Streit mit Korsching auf sein Zimmer gegangen war, hörte man ihn schluchzen. Die englischen Redakteure der Farm-Hall-Protokolle bemerkten dazu, dass er sich »in der Lage eines besiegten Generals « zu sehen schien; siehe ebd., S. 156. 
unverständliche Formeln hingeschrieben. Wir haben Experimente nicht mit genügendem Nachdruck betrieben $« .64$

Bagge war wie von Weizsäcker ein Schüler Heisenbergs; er promovierte 1938 bei Heisenberg (fünf Jahre nach von Weizsäcker) und wurde in Leipzig anschließend Heisenbergs Assistent (ebenfalls nach von Weizsäcker). Auf Antrag Diebners wurde Bagge am 8. September 1939 durch einen militärischen Gestellungsbefehl an das HWA in Berlin beordert. ${ }^{65}$ Kurz darauf, im Oktober 1939, kam Bagge als wissenschaftlicher Mitarbeiter an das Kaiser-Wilhelm-Institut für Physik (KWIP) in Dahlem. Dort begann er an einer experimentellen Vorrichtung zur Isotopentrennung zu arbeiten. ${ }^{66}$ Rückblickend notierte er in seinem Tagebuch am 7. August 1945:

"Die Amerikaner und Engländer haben in Amerika gewaltige Fabriken aufgezogen und ungestört in pausenloser Arbeit das reine Uran 235 hergestellt. Währenddessen mußten wir in Deutschland um ein paar tausend Mark riesige Kämpfe ausfechten und zusehen, wie unsere Arbeiten immer wieder zerbombt wurden, mußten freilich auch feststellen, wie einige unserer maßgebenden Männer die Isotopentrennung abschlägig beurteilten und sie nur so am Rande duldeten ${ }^{6}{ }^{67}$

Dieses Tagebuch erschien im Mai 1957 mit einer längeren historischen Einleitung Bagges und Diebners. Die Göttinger Erklärung vom 12. April, die sich gegen Konrad Adenauers und Franz Josef Strauss' atomare Bewaffnungspläne richtete, wurde weit und breit zur Kenntnis genommen, ${ }^{68}$ während der Abriss »Zur Entwicklung der Kernenergie-Verwertung in Deutschland « von Bagge und Diebner ohne vergleichbare öffentliche Resonanz blieb, obwohl oder gerade weil er die Kriegsjahre einschloss und hervorhob, dass man in Deutschland seit Mitte 1940 »von der Realisierbarkeit einer Atombombe wußte und daß man sowohl das Uran 235 als auch das Element 94 als Sprengstoff benutzen kann « ${ }^{69} \mathrm{Im}$ Anschluss an diese zutreffende Feststellung schrieben Bagge und Diebner:

"Unter dem Eindruck dieser Möglichkeit machten sich einige maßgebende deutsche Physiker den Standpunkt zu eigen, es sei nicht nötig, die direkte Isotopentrennung besonders zu fördern und zu beschleunigen. Nach dem Anlauf der Uranbrenner stehe bald ausreichend Plutonium zur Verfügung, das praktisch als Ersatz für das Uran 235 betrachtet werden könne. Wenn diese Einstellung auch nicht zu einer Stillegung aller Versuche zur Isotopentrennung beim Uran führte, so bedeutete sie für die an dieser Aufgabe Arbeitenden [Bagge, Harteck und Korsching] zumindest eine gewisse Lähmung «. ${ }^{70}$

64 Ebd., S. 159.

65 Bagge et al. 1957, S. 22.

66 Siehe Karlsch 2005, S. 62: »Am Jahresende 1941 begannen die Arbeiten zum Bau der Isotopenschleuse in der Werkstatt des KWI für Physik «. Bagges »Isotopenschleuse « produzierte im August 1944 nach 120 Stunden ununterbrochener Operation zweieinhalb Gramm angereichertes Uranhexafluorid; siehe auch Walker 1990 a, S. 164.

67 Bagge et al. 1957, S. 57.

68 Siehe Kraus 2001, S. 386-388, für eine Bibliographie der »Zeitgenössische[n] Presse im Kontext der Göttinger Erklärung «.

69 Ebd., S. 39.

70 Ebd., S. $39 \mathrm{f}$.

Leviathan, 41. Jg., 3/2013 
Schon in der Nacht vom 6. auf den 7. August 1945 hatte Bagge zu Diebner gesagt, dass Heisenberg »nicht der richtige Mann dafür war « und von Weizsäcker ebensowenig; dass Korsching »mit dem, was er zu Gerlach äußerte, recht hat[te]«; und dass er [Bagge] seine Vorrichtung zur Isotopentrennung "gegen Heisenbergs Wunsch « entwickeln musste. ${ }^{71} 1957$ beschuldigten Diebner und Bagge die »maßgebenden deutschen Physiker « - Heisenberg und von Weizsäcker, die den Uranverein dominiert hatten und nun wieder an der Spitze standen -, den deutschen Rückstand in der Uranisotopentrennung (und damit das Misslingen des Baus einer deutschen Atombombe) verursacht zu haben.

Bagge und Diebner ließen in ihrer Bemerkung über die Produktion von Plutonium erkennen, dass die Geschichte der deutschen nuklearen Zurückhaltung eine Legende war. Die Propagierung des » friedlichen « Uranbrenners, der nur Energie und keine Kernsprengstoffe liefert, war für alle Eingeweihten professioneller Unsinn zum Zwecke der Verschleierung der militärischen Bedeutung einer Uranmaschine. ${ }^{72}$ Mit der Behauptung, dass Heisenberg und von Weizsäcker die Isotopentrennung vor allem darum behindert hatten, weil sie auf die Erzeugung von waffenfähigem Plutonium in der Uranmaschine setzten, gaben Bagge und Diebner dreierlei zu verstehen: (i) dass alle Beteiligten am deutschen Uranprojekt im Endeffekt auf die Herstellung einer Atombombe hinarbeiteten, (ii) dass alternative Wege zu diesem Ziel führten und verfolgt wurden sowie (iii) dass Heisenberg und von Weizsäcker im Interesse des Reaktorbaus den Weg der Uranisotopentrennung marginalisierten. Gegen diese drei Punkte sind keine historisch-faktischen Einwände zu erheben.

Heisenberg und von Weizsäcker haben Bagge und Diebner ebenfalls nicht widersprochen, und sie mussten das auch gar nicht. Ihre »Nachkriegsapologetik «73 bestand darin, Angriffe dieser Art dadurch zu entwerten, dass sie politisch kompromittierte Kollegen wie Diebner nicht nur ins Schattenreich der politisch »ewig Gestrigen " abdrängten, was durchaus berechtigt und notwendig war, sondern auch als "Nichtskönner «74 disqualifizierten, was keinesfalls zutraf.

Schon Goudsmit war die deutsche Hackordnung aufgefallen, als er in Hechingen nicht nur Hahn, von Laue, von Weizsäcker und Wirtz internierte, sondern auch » two of the younger men, because they had done some novel work on isotope separation «. Er bemerkte: »This puzzled von Weizsäcker very much; he evidently thought that the young fellows were not important enough to be interned. ' What

71 Hoffmann 1993 b, S. 170.

72 Vgl. von Weizsäckers Patentanmeldung, S. 3: »Die Erzeugung des Elements 94 [Plutonium] in praktisch brauchbarer Menge ist am besten in der $>$ Uranmaschine $<$ möglich. [...] Ganz besonders vorteilhaft ist es - und das bildet den Hauptgewinn der Erfindung - daß das entstandene Element 94 leicht chemisch [...] von dem Uran getrennt und rein dargestellt werden kann« (zum Fundort siehe Anmerkung 86).

73 Walker 1990 a, S. 247.

74 Ebd., S. 246. Diese Strategie des Uranvereins hat Walker auf folgende Kurzformel gebracht: "Während des Dritten Reichs waren alle 'guten`, ‘fähigen`Wissenschaftler > unpolitisch`, während umgekehrt alle `politischen`Wissenschaftler weder gut noch fähig waren« (ebd., S. 250). 
kind of selection is this! he complained $« .{ }^{75}$ Die Tatsache, dass von Weizsäcker und die »zwei jüngeren Männer « - Bagge und Korsching - allesamt Jahrgang 1912 waren, hatte Goudsmit bezeichnenderweise »vergessen «. ${ }^{76}$ Als ein »Herr Professor Doktor « war von Weizsäcker auch für Goudsmit »älter " als von Weizsäckers gleichaltrige Kollegen, die beide nur Doktoren waren. 1957 hatten Bagge und Diebner sicher große, lang aufgestaute Lust, aber keine Chance mehr, den zivilen Atomkurs und -diskurs zu torpedieren, den von Weizsäcker und Heisenberg erfolgreich vorantrieben. ${ }^{77}$

Die antagonistische Dynamik im deutschen Uranprojekt durchzieht die FarmHall-Protokolle umso mehr, als die Internierten seit Hiroshima damit beschäftigt waren, durch Schuldzuweisungen an externe und interne Gegner mit der existierenden amerikanischen und der nichtexistierenden deutschen Atombombe zurechtzukommen. Wirtz, der mit von Weizsäcker in Farm Hall das Zimmer teilte, reagierte auf die Auseinandersetzung zwischen Korsching und Gerlach mit der Anerkennung des Konflikts in der »Urangruppe «: »Korsching hatte wirklich recht, als er sagte, es habe in der Urangruppe keine sehr gute Zusammenarbeit gegeben, wie Gerlach behauptet hat. Gerlach hat eigentlich gegen uns gearbeitet. Er und Diebner haben die ganze Zeit gegen uns gearbeitet ${ }^{78}$ Wirtz beschrieb damit die Gruppenperspektive des Uranvereins. Was Gerlach wirklich getan hatte, war, wozu er von Amts wegen verpflichtet gewesen war, nämlich das gesamte Uranprojekt voranzutreiben. ${ }^{79}$ Und das gesamte Projekt beschränkte sich nicht allein auf die Arbeiten des Uranvereins, sondern umfasste auch die mit dem HWA eng verbundenen Aktivitäten der Diebner'schen Forschungsgruppe, die in der »Versuchsstelle Gottow « südlich von Berlin, am Rande der großen Heeresversuchsanstalt Kummersdorf, ther-

75 Goudsmit 1947, S. 104.

762012 wurde der 100. Geburtstag von Weizsäckers im deutschsprachigen Raum mit vielen Artikeln, Veranstaltungen und Konferenzen gefeiert, ohne ein Aufheben von Bagge und Korsching zu machen.

77 Die Intervention von Bagge und Diebner hatte historische Brisanz. Ihre »Bitterkeit «, dass »die Verwertung der in den Atomkernen schlummernden Energievorräte ausgerechnet in Deutschland noch immer nicht realisiert werden konnte " (Bagge et al. 1957, S. 9), war hauptsächlich von persönlicher Bedeutung. Und ihre Zukunftsperspektive, die auf dem Buchumschlag mit einem Foto des englischen Atomkraftwerks Calder Hall (welches Elektrizität und Plutonium produzierte) angedeutet wurde, war von der Göttinger Erklärung der alten Gegenspieler glücklicherweise sowohl eingeholt als auch überholt worden.

78 Hoffmann 1993 b, S. 165.

79 Siehe Walker 2007, S. 383: »Gerlach gelang der Spagat, Diebner und Heisenberg gleichzeitig zu unterstützen «. 
monukleare Sprengversuche mit Hohlkörpern anstellte und zunehmend erfolgreichere Versuche mit Modellreaktoren unternahm. ${ }^{80}$

Die Relativierung der Vormachtstellung des Uranvereins ist ein verspätetes Ergebnis der Wissenschaftsgeschichte, die allzu oft die Brecht'sche Erkenntnis bestätigte: "Doch man sieht nur die im Lichte, die im Dunklen sieht man nicht «. Schon seit David Irvings Buch ${ }^{81}$ war bekannt, dass Diebners Gottower Experimente den Uranverein in Sachen Brennerarchitektur und Neutronenvermehrung zwischen Frühjahr 1943 und 1944 überflügelt hatten, ${ }^{82}$ doch der spätere Reputationsverlust des Autors tabuisierte diesen wichtigen Beitrag im Nachhinein. Gegen die habituelle Unterschätzung der »Dunkelmänner « machte Rainer Karlsch im Jahre 2005 mit Hitlers Bombe Front. Sein Buch zeigte, dass »Die Anderen ${ }^{83}$ um Diebner bis zuletzt auf den Bau einer deutschen Atomwaffe hingearbeitet und mit einer waffentechnischen Alternative zur Uran- und Plutoniumbombe experimentiert hatten. ${ }^{84}$ Harte Belege für die These, der zufolge Diebners Gruppe im März 1945 eine »taktische Kernwaffe « 85 getestet habe, konnte Karlsch nicht beibringen, dafür entdeckte er jedoch eine Reihe bislang unbekannter Dokumente, von denen eines die keineswegs friedlichen Forschungsabsichten des Uranvereins zeigt: von Weizsäckers »Patentansprüche zu nuklearen »Verfahren « der Energiegewinnung und der Erzeugung einer Plutoniumbombe. ${ }^{86}$

Die Frage bleibt, warum sich von Weizsäcker und Wirtz gegen Diebner und Bagge stellten, wenn doch grundsätzlich Einigkeit darüber bestand, dass Deutschland falls wissenschaftlich-technisch überhaupt möglich - eine Atomwaffe besitzen sollte

$80 \mathrm{Zu}$ Diebners drei Gottower »Großversuchen « mit Modellreaktoren vgl. Günter Nagels (2002) wegweisende regionalgeschichtliche Arbeit. Karlsch konnte auf Nagel mit der Entdeckung eines vierten Versuchs aufbauen; siehe Karlsch 2005, S. 132. Wie in der Wissenschaft, so auch in der Wissenschaftsgeschichte: Für innovative Anstöße müssen manchmal die "Außenseiter « sorgen (hier der Jurist Nagel und der Wirtschaftshistoriker Karlsch).

81 Irving 1967 a; Irving 1967 b. Ein Vorabdruck erschien im Spiegel; siehe Irving 1967 c. Irvings Entwicklung zum notorischen Holocaustleugner, Rassisten und Antisemiten begann 1977, zehn Jahre nach der Publikation seiner auf Englisch verfassten Pionierarbeit.

82 Siehe Irving 1967 b, S. 270 ff.; Walker 1990 a, S. 118-131.

83 Karlsch 2005, S. 43.

84 Siehe hierzu auch Karlsch, Walker 2005.

85 Karlsch 2005, S. 24, 219. In einem Folgeband hat Karlsch zu präzisieren versucht, wovon »Hitlers Bombe« handelte: »Wir gehen davon aus, dass deutsche Wissenschaftler [unter Anleitung von Gerlach und Diebner] eine Hybridanordnung, bestehend aus viel Sprengstoff und einer kleinen Menge Spalt- und Fusionsstoff, entwickelt hatten und diese im März 1945 erfolgreich testeten. 'Erfolgreich ‘ heißt in diesem Fall eine Explosion mit Freisetzung von kinetischer Energie und Radioaktivität «; siehe Karlsch, Petermann 2007, S. 19.

86 Karlsch 2005, S. 73-76, 322-324 (Auszug, Faksimile). Das vollständige Dokument ist im Archiv der Max-Planck-Gesellschaft zu finden (I. Abt., Rep. 34 KWI für Physik, Nr. 73/4). 
und alle beteiligten Gruppen dieses Ziel zumindest anfänglich uneingeschränkt teilten.

Die Ausweitung der historischen Perspektive auf die Systemfunktionäre Gerlach und Diebner sowie die einschlägigen technischen Gruppen des Militärs, der SS und des Reichspostministers begründete Karlsch mit der Feststellung, dass Diebner der »eigentliche Organisator des Kernforschungsprojektes « ${ }^{87}$ gewesen sei. Richtig an dieser Äußerung ist, dass Erich Schumann, der Direktor der Forschungsabteilung des HWA, Diebner im Juni 1939 mit der Leitung des neuen Referats für Atomphysik beauftragte und ihn nach der kriegsbegründeten Übernahme des KWIP durch das HWA zum kommissarischen Direktor des Eliteinstituts machte. So kam es, dass Diebner in Gottow (danach dem Ausweichquartier Stadtilm) federführend war und von 1940 bis 1942 von Weizsäcker, Wirtz und allen anderen Mitarbeitern des KWIP vorstand. Falsch wäre jedoch die Annahme, dass Diebner die Zügel des deutschen Uranprojekts fest in der Hand gehabt hätte. Diebner musste nicht nur mit den wechselnden Schwierigkeiten am Ende einer polykratischen Vorgesetzten- und Kommandokette zurechtkommen, sondern auch im Gehege der deutschen Ordinarien- und Koryphäennetze operieren. In Gottow konnte er mit jungen Wissenschaftlern arbeiten, die ihn respektierten und aufs Dritte Reich eingeschworen waren; ${ }^{88}$ in Berlin konnte er nur auf Bagge bauen. Insgesamt musste er mit der Geringschätzung und dem Widerstand seitens der akademischen Elite kämpfen.

Die Gründe für Diebners schweren Stand gegenüber den akademischen Würdenträgern waren nicht die, die man sich in erster Linie wünschen würde. Den Ausschlag gaben weder die Parteizugehörigkeit Diebners, der nach von Weizsäckers Einschätzung "nicht einmal ein aufrichtiger Nazi ${ }^{89}$ war, noch die Requirierung des KWIP. ${ }^{90}$ Dass dessen holländischer Direktor, der Nobelpreisträger Peter Debye, mit der Alternative konfrontiert wurde, entweder die deutsche Staatsangehörigkeit anzunehmen oder die Leitung des Instituts abzugeben, war auch nicht entscheidend. ${ }^{91}$ Der Stein des Anstoßes war vielmehr, dass Diebner mangels Habilitation $^{92}$ nicht den Anforderungen entsprach, die auch im Hitlerreich an einen deutschen Professor gestellt wurden, geschweige denn die Voraussetzungen erfüllte, die für Institutsdirektoren der KWG galten. ${ }^{93}$ Für zwei wissenschaftliche Mitarbeiter des KWIP genügte das, sich gegen Diebner zu »verschwören «, um Heisenberg ins

87 Ebd., S. 106.

88 Ebd., S. 43: »Alle seine neuen Mitarbeiter waren Angehörige der NSDAP oder einer ihrer Gliederungen «

89 Weizsäcker 1993, S. 343.

90 Zur Übernahme des KWIP durch die Reichsregierung und »kampflosen Preisgabe des Instituts « von Seiten der Kaiser-Wilhelm-Gesellschaft siehe Walker 1990 a, S. 32 f.

91 Siehe Kant 2011 zur »Debye-Affäre.

92 Gerlach versuchte, Diebner von diesem Makel zu befreien; siehe Nagel 2002, S. 93.

93 Siehe Walker 2007, S. 356.

Leviathan, 41. Jg., 3/2013 
Spiel zu bringen. Mit Behagen hat noch 1993 von Weizsäcker diese »kleine taktische Geschichte« erzählt:

»Eines Tages - Diebner war unser Chef geworden, ich nehme an das war 40 - kommt Wirtz zu mir und sagt: >Hör mal, wir müssen den Diebner loswerden. Da gibt es eine Möglichkeit. Wir könnten doch zusehen, ob wir nicht Heisenberg zum Direktor des Instituts machen. Dann sind wir Diebner los. Wir redeten hin und her und kamen zu der Folgerung: Wir müssen zuerst Diebner bewegen, daß er es zu Heisenbergs Pflichten macht, jede Woche mehrere Arbeitstage im Institut zu sein «. ${ }^{94}$

Dazu kam es dann auch, freilich nicht zuletzt deswegen, weil Bagge schon im September 1939 Diebner vorgeschlagen hatte, Heisenberg in den Arbeitskreis der Kettenreaktionsforscher des HWA aufzunehmen. ${ }^{95}$ Diebner machte Heisenberg zum »Berater der Berliner Gruppe « ${ }^{96}$ (per Gestellungsbefehl), was dazu führte, dass Heisenberg ab Oktober 1940 regelmäßig zwischen den Uranarbeiten an seinem Leipziger Lehrstuhl ${ }^{97}$ und am KWIP zu pendeln begann. ${ }^{98}$ Ein gutes Jahr später ging von Weizsäcker »im Einverständnis mit Wirtz und natürlich auch mit Heisenberg « zu den Dahlemer KWI-Direktoren Hahn (Chemie), Alfred Kühn (Biologie) ${ }^{99}$ und Adolf Butenandt (Biochemie) und unterbreitete ihnen den Vorschlag der »Verschwörer « zur Ablösung Diebners:

»)Hören Sie, es gibt jetzt die Chance, daß wir Heisenberg zum Direktor machen. Würde die Kaiser-Wilhelm-Gesellschaft das wollen? Und die sagten ja, großartiger Gedanke, haben das dann miteinander in der KWG vorgebracht und durchgesetzt, und dann plötzlich war klar, daß Diebner weg ist. Erst da hat Diebner möglicherweise verstanden, daß das eine Verschwörung war, vielleicht hat er es auch nie verstanden «. ${ }^{100}$

Das ist zu bezweifeln. Diebner, der sich, was selbst von Weizsäcker einräumte, zumindest »in der Verwaltung auskannte « ${ }^{101}$ verstand die Winkelzüge der Mitarbeiter vermutlich besser als ihm lieb war, passten sie doch gänzlich in die von Diebner erfahrene und beklagte »'patriarchalische< Verfassung der deutschen Forschungsinstitute für Physik «. ${ }^{102}$ Die Herabsetzung Diebners im Doppelspiel von Weizsäckers und Wirtz' war typisch für diese Verfassung; ausschlaggebend für Diebners Ablösung war die Intrige der beiden Mitarbeiter jedoch überhaupt nicht - was von Weizsäcker wohl wusste, aber nur mit den kryptischen Worten »es gibt jetzt die Chance« andeutete.

Die Möglichkeit, die von Weizsäcker erspäht hatte, ergab sich aus der seit Anfang Dezember 1941 vom HWA anvisierten Abstoßung des Uranprojekts (mangels

94 Weizsäcker 1993, S. 342 f.

95 Siehe Bagge et al. 1957, S. 23; Walker 1990 a, S. 32.

96 Kant 1993, S. 153.

97 Siehe Kleint 1993.

98 Siehe Cassidy 1992, S. 427.

99 In einer Erinnerung von 1987 suchte von Weizsäcker nicht Kühn, sondern Fritz von Wettstein am KWI für Biologie auf; siehe Weizsäcker, Hora 2002, S. 216.

100 Weizsäcker 1993, S. 343.

101 Ebd.

102 Bagge et al. 1957, S. 13. 
unmittelbarer Kriegsrelevanz) an eine mit der Grundlagenforschung beschäftigte Organisation, den RFR ${ }^{103}$ oder die KWG. ${ }^{104}$ Kurz vor Weihnachten 1941 hatte Schumann in einem Arbeitstreffen mit Hahn und anderen Projektleitern diese Eventualität erwähnt und nicht nur das ungelöste Problem der Debye-Nachfolge am KWIP angeschnitten, sondern auch den Experimentalphysiker Bothe als besten Nachfolgekandidaten ins Gespräch gebracht. Die Wirtz-von Weizsäcker'sche Intervention für Heisenberg richtete sich daher nicht vorrangig gegen Diebner, sondern gegen Bothe, der mehreren KWG-Direktoren und -Projektleitern in persönlicher und fachlicher Hinsicht weniger genehm als Heisenberg war. ${ }^{105}$ Diebner verlor seine Stellung am KWIP also nicht, weil Heisenberg berufen wurde, sondern weil Schumann beschlossen hatte, die Kontrolle über das Uranprojekt abzugeben.

Mit dem Hereinholen Heisenbergs endete von Weizsäckers Verschwörung wie gewünscht, doch nicht wie geplant. Im März 1942 war es dem RFR gelungen, die KWG » auszustechen « und ihr das Uranprojekt » wegzunehmen «. ${ }^{106}$ Die Verhinderung der Berufung Bothes im April 1942 war der erste Schritt des RFR nach dem Beginn der »feindlichen Übernahme « des Projekts. ${ }^{107}$ Der zweite folgte Anfang Juli mit der Ernennung Heisenbergs zum Direktor am KWIP. ${ }^{108}$ Mit der Abkommandierung Diebners und seiner Gottower Gruppe an die Physikalisch-Technische Reichsanstalt (PTR) im März 1943 besiegelte der dritte die Entbindung des HWA aus der Projektverantwortung. ${ }^{109}$ Präsident der PTR, die zum Reichserziehungsministerium (REM) gehörte, war Abraham Esau, der gleichzeitig die RFR-Fachsparte für Physik leitete. Göring, dem seit Juni 1942 auch der RFR unterstand, ernannte Esau im Dezember 1942 zum »Bevollmächtigten für alle Fragen der Kernphysik « ${ }^{110}$ Mit dieser Beförderung trat Esau nach dem »Rückzug «111 des HWA aus der Atomforschung an die prekäre Stelle, die Schumann vorher eingenommen hatte und Gerlach später einnehmen sollte: die Verwaltung der antagonistischen Gruppendynamik im deutschen Atomprojekt.

103 Siehe ebd., S. 28.

104 Siehe Walker 1990 a, S. 66.

105 Ebd., S. 78.

106 Ebd., S. 77.

107 Ebd., S. 79.

108 Hentschel 1996, S. XXXI. Dass der Senat der KWG Heisenberg nicht als Direktor des KWIP, sondern zum »Direktor am KWI für Physik " (Kant 2011, S. 104) berief, ergab sich aus dem Umstand, dass der offizielle Direktor (Debye) formal lediglich »beurlaubt « war. 1943 wurde die Entscheidung über Debyes Position » bis nach Kriegsende zurückgestellt« (Rispens 2011, S. 75).

109 Nagel 2002, S. 81.

110 Ebd., S. 80, 323; siehe auch Walker 1990 a, S. 112.

111 Der größere organisationspolitische Zusammenhang, in den der »Rückzug « des HWA nach dem Amtsantritt von Albert Speer am 8. Februar 1942 als Reichsminister für Bewaffnung und Munition zu stellen ist und der im März 1942 durch ein "generelles >Verbot des Konstruierens im Heereswaffenamt « ausgesprochen « (Ciesla 2007, S. 68) wurde, ist hier zu berücksichtigen. 
Bagge und Diebner erlebten den Verlust der Rückendeckung durch das HWA als die »entscheidende Wende « ${ }^{112}$ und kommentierten den Rückschlag 1957 mit Sarkasmus, Enttäuschung und Kritik:

"So wurde denn die ganze Organisation einem Hochfrequenztechniker, nämlich Prof. Dr. Esau, übertragen, der im Auftrage des Reichsforschungsrates die Arbeiten weiterzuleiten hatte. Damit war die Schlagkraft dieser Organisation weitgehend gelähmt. Ihr fehlten von nun an die machtpolitischen Möglichkeiten des Oberkommandos des Heeres «. ${ }^{113}$

Nach der »Kleinschreibung « des deutschen Atomwaffenprojekts drohte für von Weizsäcker das Ende der Unabkömmlichkeitseinstufung. Diese Konsequenz der größeren »taktischen Geschichte«, die ihn und Harteck im Januar 1942 gefährlich nahe an den Fronteinsatz in Russland brachten, erwähnte von Weizsäckers »kleine taktische Geschichte 1993 nicht. ${ }^{114}$ Es ist daher zu betonen, dass die Herabstufung der deutschen Uranarbeiten auf das Niveau der Grundlagenforschung eine Entscheidung der Wissenschaftsbevollmächtigten des Regimes und nicht der Wissenschaftler war (was von Weizsäcker und Heisenberg oft verwischten). Insgesamt gilt, dass die Rivalen im deutschen Uranprojekt über ihre wichtigsten historischen Beiträge - Diebners thermonukleare Experimente und von Weizsäckers Bombenpatentanmeldung - trotz aller Worte, die sie der Vergangenheit widmeten, gleichermaßen schwiegen.

\section{Patentansprüche: von Weizsäckers »Uranbomben-Geschichte«}

»Und als dann die Uranbomben-Geschichte anfing, hatte ich das Gefühl, es ist ja nicht völlig ausgeschlossen, daß es mir zufällt, derjenige zu sein, der Hitler gerade noch davor rettet, sich selber umzubringen, damit er das ermöglicht, was er eigentlich ermöglichen sollte. «115

Carl Friedrich von Weizsäcker, 3. Juni 1993

Das, was Hitler »eigentlich ermöglichen sollte ", ist gegenwärtig in Ermangelung privater Quellen und infolge der sprachlichen Absicherung von Weizsäckers im öffentlichen Diskurs gegen alles Eindeutige noch nicht mit Bestimmtheit zu sagen. 1943 kam es jedoch zu einem unkontrollierten Ausbruch unter vier Augen. In einer heftigen Auseinandersetzung mit Heisenberg, die am 13. Oktober stattfand und am 14. Oktober in einem ausführlichen Brief Heisenbergs an seine Frau Elisabeth geschildert wurde, vertrat von Weizsäcker extrem radikale und eminent erklärungs-

112 Bagge et al. 1957, S. 27.

113 Ebd., S. 29.

114 Siehe Walker 1990 a, S. 64: »In beiden Fällen war es Werner Heisenberg, dem es aufgrund seiner engen Beziehungen zu führenden Militärs mit viel Mühe gelang, Erich Schumann und das Heereswaffenamt umzustimmen und eine Verlängerung der U.k.Stellung für Harteck und von Weizsäcker zu erwirken «.

115 Weizsäcker 1993, S. 339. 
bedürftige Ansichten. ${ }^{116}$ Den Ursprung dieser Ansichten habe ich in der Verbindung von Weizsäckers mit Martin Heidegger gefunden. ${ }^{117}$ Der junge von Weizsäcker teilte das essentialgeschichtliche Denken, mit dem der Freiburger Philosoph den real existierenden Nationalsozialismus sowohl kritisierte als auch überbot. Ich nehme an, dass von Weizsäcker Hitlers weltgeschichtliche »Aufgabe « in der Errichtung des wabren Dritten Reichs sah, das heißt in der Erfüllung der Vision des "utopischen " Nationalsozialismus Heidegger'scher Prägung.

Wann »die Uranbomben-Geschichte anfing ", ist seit 1967 bekannt: im Sommer 1940. Schon Irving hatte auf von Weizsäckers einschlägigen Geheimbericht (G-59)118 — »Eine Möglichkeit der Energiegewinnung aus 238U « vom 17. Juli 1940 hingewiesen. ${ }^{119}$ Der Bericht hob die Eignung eines trans-uranischen Elements (Plutonium) als »Sprengstoff « klar hervor und ging, wie hunderte von Berichten dieser Art, selbstverständlich über Diebner an das HWA. ${ }^{120}$ Die bundesrepublikanische Wahrnehmung dieser beiden Tatsachen war jedoch schleppend. Ein SpiegelArtikel »Übers Plutonium kann man was machen «, der sich auf Walkers Dissertation (Princeton 1987) stützen konnte, beförderte die Rezeption zum 50. Jahrestag der Entdeckung der Kernspaltung mit der seit Jungk kursierenden Frage: »Konnten und wollten die deutschen Wissenschaftler - womöglich noch vor den Amerikanern - die kriegsentscheidende Waffe bauen? Oder leisteten sie bewußt hinhaltenden Widerstand? "121 Das Ende der Unschuld, ein zweiteiliges Fernsehspiel von Wolfgang Menge »über die Deutschen und ihre Atombombe «, ${ }^{122}$ sorgte im Frühjahr 1991 erneut für Aufsehen. Von Weizsäcker sah den zweiten Teil im gesamtdeutschen Zusammenhang des Jahrestreffens der Leopoldina in Halle. ${ }^{123}$ Er nahm Stellung und erklärte die These des Films für »massiv falsch «. ${ }^{124}$ Daraufhin richtete Der Spiegel die Frage an von Weizsäcker: »Konnten oder wollten die deutschen Wissenschaftler nicht? «125

116 Siehe Hirsch-Heisenberg 2011, S. 224 f.

117 Siehe Schäfer 2013.

118 Weizsäcker 1940.

119 Irving 1967 b, S. 75, 315.

120 Für eine Liste der deutschen Geheimberichte (G-Reports) siehe Walker $1990 \mathrm{a}$, S. 313-319.

121 SPIEGEL 1988, S. 152. Walkers Dissertation wurde 1989 publiziert; siehe Walker 1989.

122 Menge 1991.

123 Siehe www.zeit.de/1991/17/abend-der-physiker-ueber-das-ende-der-unschuld (Zugriff vom 19. Mai 2013).

124 Weizsäcker 1991 a: »Es ist die These, die Deutschen hätten, wenn sie gewisse technische Fehlentscheidungen vermieden hätten, die Atombombe während des Krieges und schon früher als die Amerikaner bauen können «. Ebd.: »Etwa damals [Ende 1941] entschloß sich die amerikanische Gruppe definitiv zur Bombe, die deutsche Gruppe definitiv, nicht an der Bombe zu arbeiten, sondern nur an Reaktoren «.

125 Weizsäcker 1991 b, S. 227. 
An dieser Stelle ist zeitgeschichtlich innezuhalten. Von Weizsäckers individueller Lernprozess, der in der Göttinger Erklärung seinen Abschluss fand, wurde gut 30 Jahre später, am Vorabend der deutschen Wiedervereinigung, vom gesellschaftlichen Lernprozess »eingeholt «. Was kann, was soll von Weizsäcker in diesem Moment tun? Er ist dem sozialen Lernprozess vorausgeeilt und im Besitz des gefragten historischen Wissens; er hat sich mit Blick auf die Zukunft vor über vier Jahrzehnten dagegen entschieden, über die Vergangenheit zu reden; jetzt tritt die Gegenwart dieser Zukunft an ihn heran und verlangt von dem bald 80-Jährigen die Antworten, die er sich selbst gab, mit denen er sich nun aber, wenn er sie nach so langer Zeit der Zurückhaltung öffentlich machen würde, nur doppelt beschuldigen kann. Von Weizsäcker traf seine Wahl; er behielt den einmal eingeschlagenen Kurs bei.

Auf die Frage der Spiegel-Redakteure »Und was hat Sie motiviert? « gab von Weizsäcker eine dreiteilige Antwort: (i) das eingangs erwähnte generelle Schuldbekenntnis (» Was ich damals gemacht habe, gehört zu denjenigen Handlungen meines Lebens, die ich nachträglich nicht noch einmal wiederholen würde. Ich habe falsch gehandelt. «); (ii) eine Erklärung seines Desinteresses an der Technik (»Das Technische interessierte mich überhaupt nicht, wissenschaftlich fand ich andere Sachen viel interessanter. «); und (iii) die Idee, mit seinem wissenschaftlich-technischen Knowhow politisches Kapital zu erringen (»Ich hatte die Vorstellung, daß ich politischen Einfluß gewinnen könnte, wenn ich jemand wäre, mit dem selbst Adolf Hitler reden müsste. «). ${ }^{126}$ Gebeten, diesen Gedanken zu erläutern, erklärte von Weizsäcker: »Ich habe geglaubt - das war mein großer Irrtum -, es könnte ja sein, daß man den Hitler zu einer Politik des Friedens bewegen kann. Dazu können Sie natürlich sagen, ich sei verrückt gewesen. Und ich bin bereit zuzugeben: Ich war verrückt «.127

Die Journalisten quittierten von Weizsäckers Angebot, den jungen von Weizsäcker und seine friedenspolitische Hoffnung für »verrückt « à la Don Quichotte zu erklären, mit der sarkastischen Replik »Also haben Sie Friedenspolitik machen wollen, als Sie im Juli 1940 das Heereswaffenamt über Ihre Entdeckung informierten, daß mit dem Zerfallsprodukt Plutonium Atomsprengkörper hergestellt werden können «. ${ }^{128}$ Woraufhin von Weizsäcker das Machtkalkül des damals 28-Jährigen näher beschrieb:

»Ich hatte einfach überlegt, daß es möglich ist, mit den Folgeprodukten des Urans, die man heute als Plutonium bezeichnet, leicht eine Spaltung vorzunehmen. Es müßte also möglich sein, das, was man sonst nur mit Uran 235 kann, weil es mit 238 nicht geht, auch mit Plutonium zu machen. Dann war die Frage: Soll ich das für mich behalten oder darüber einen Bericht schreiben? Ich habe einen Bericht geschrieben, den habe ich dann auch abgeliefert. Wenn Sie mich jetzt fragen, was für einen Grund das hatte, dann antworte ich: Ich dachte, wenn der Bau einer Bombe auf diese Weise möglich ist, dann werde ich einer sein, mit dem man darüber reden muß. Und dann werde ich zusehen, daß ich einen Weg zu den

126 Ebd., S. 232.

127 Ebd., S. 234.

128 Ebd. 
wirklichen Entscheidungsträgern finde, um mit denen etwas zu bereden, was diese unteren Burschen sowieso nicht verstehen ${ }^{129}$

Nun wollten von Weizsäckers Gesprächspartner natürlich genauer wissen, was das denn hätte sein können. Von Weizsäcker erklärte: »Ich dachte, wenn ich mit Hitler rede, dann werde ich ihm sagen, was ihm all seine Unterlinge nicht vortragen. Und dann wollen wir mal sehen, ob dieser Hitler, der zwar ein schrecklicher Mann, aber auch hochbegabt ist, nicht darauf anspringt «. ${ }^{130}$ Damit »verriet « von Weizsäcker abermals nur, dass er gerne mit Hitler geredet hätte, nicht aber, was er Hitler konkret vortragen wollte. Obwohl von Weizsäcker die Vermutung suggerierte, dass es schlicht die » verrückte Idee « war, die Plutoniumbombe dem » hochbegabten « Hitler für ein friedenspolitisches Ziel anzubieten, müssen wir fragen, wie das in den historischen Kontext von 1940/41 passt. Worauf sollte der Führer in von Weizsäckers Machttraum »anspringen «?

Die Plutonium-Alternative zur Uranisotopentrennung wurde 1940 auf beiden Seiten des Atlantiks erkannt. Sie war eine klassische multiple discovery, die über einige Schritte (mit anfänglichen Irrtümern) in wenigen Monaten zustande kam. ${ }^{131}$ Von Weizsäckers Bericht und ein Memorandum des Berkeley-Physikers Ernest Lawrence vom Mai 1941 ähnelten sich dermaßen in ihren hauptsächlichen Schlussfolgerungen (»sehr kleine Maschinen « und ein Sprengstoff, der eine »super bomb « ermöglichen würde ${ }^{132}$ ), dass beide Darstellungen "praktisch eine Übersetzung des jeweils anderen Textes « ${ }^{133}$ waren. Wesentliche Unterschiede zwischen Deutschland und den Vereinigten Staaten bestanden ursprünglich darin, dass Deutschland seit dem 1. September 1939 Krieg führte und seitdem auch ein staatlich gefördertes und organisiertes Atomprojekt unter HWA-Regie hatte, während die USA erst nahezu zwei Jahre später die organisatorischen Voraussetzungen für das spätere Manhattan-Projekt schufen ${ }^{134}$ und auch erst nach dem japanischen Angriff auf Pearl Harbor, der am 7. Dezember 1941 erfolgte, in den Zweiten Weltkrieg eintraten.

Dass Hitlers Blitzkriege in den beiden ersten Kriegsjahren erfolgreich für Deutschland verliefen, war von entscheidender Bedeutung für das deutsche Situationsempfinden. Die »Siegeswelle « der Wehrmacht hatte Polen, Dänemark, Norwegen, Holland, Belgien, Luxemburg und Frankreich »ausgeschaltet «, desgleichen Jugoslawien und Griechenland. Allein Großbritannien war noch zu schlagen. Japan, Italien, Ungarn, Rumänien und Bulgarien waren mit dem Deutschen Reich verbündet. Der auf zehn Jahre abgeschlossene Hitler-Stalin-Pakt neutralisierte die Sowjetunion.

129 Ebd.

130 Ebd., S. $234 \mathrm{f}$.

131 Siehe Walker 1990 a, S. 36 f.; Karlsch 2005, S. 67 f.; Sime 2007, S. 290 f.; Brandt, Karlsch 2007.

132 Siehe Weizsäcker 1940, S. 5; Smyth 1945, S. 65.

133 Walker 1990 a, S. 205.

134 Am 28. Juni 1941 mit der Gründung des OSRD (Office of Scientific Research and Development). 
Und Präsident Roosevelt wurde von starken isolationistischen Tendenzen im amerikanischen Kongress zurückgehalten. Kurz: Im Sommer und Herbst 1941 dominierte Hitler auf der europäischen Bühne. Er hatte Deutschland die ersehnte Großmachtstellung verschafft. Für von Weizsäcker bestätigte das nur die langgehegte Vermutung, dass Hitler innen- und außenpolitisch ernst zu nehmen war. ${ }^{135}$ Der professionellen Zurückhaltung und den bürgerlichen Werten seines Vaters mit der radikalen Philosophie des utopischen Nationalsozialismus insgeheim den Rücken kehrend, begriff der junge von Weizsäcker den deutschen Führer als

» ein Instrument dessen, was er [Hitler] immer die Vorsehung nannte - ein Instrument, das selber nicht weiß, wofür es gebraucht wird. Ich war nicht überrascht, als alle Attentate auf ihn fehlschlugen. Ich hatte immer das Gefühl, so geht das mit Hitler nicht zu Ende. Ich dachte, vielleicht hat er doch irgendwo den Schlüssel für das, was kommen soll. Und mein Wunsch war zunächst einmal, in diese Sphäre der Entscheidung als Person hineinzukommen und dann zu sehen, was ich tun muß «. ${ }^{336}$

Der Plutonium-Beitrag zum deutschen Atomprojekt war von Weizsäcker offenbar nicht genug. 1941 reizten ihn zwei Pfade, der wissenschaftlich-technische zur Plutoniumbombe und der macht- und karrierepolitische mit Zugang zur Entscheidungssphäre des Führers. Nicht unbescheiden, wollte er auf beiden Pfaden vorankommen und alle um ihn herum überbieten: den Vater und die ganze skeptische Familie sowie den Doktorvater und die »unteren Burschen «, vertreten durch Diebner. Die Offerte der machbaren Atombombe war der Hebel: »Wenn ich nun eine Waffe machen kann, über die mit mir zu verhandeln niemand verhindern kann vielleicht kriege ich Einfluß auf die Ereignisse, weiß der Himmel wie «. ${ }^{137}$ Auch in dieser Version seiner Hitler-Geschichte gab von Weizsäcker nur preis, dass er mit dem Versprechen der Plutoniumbombe politisches Kapital erringen wollte, nicht aber wofür. Das »weiß der Himmel wie« deutete auf Ungeduld hin, möglicherweise Dringlichkeit, aber sowohl »die Ereignisse « als auch das Verhandlungsziel blieben unbestimmt. Wir können aus dieser Zensur schließen, dass das » Wofür« zu sensitiv

135 Siehe Weizsäcker 1988, S. 364: »Ich muß etwas zurückgreifen, in die Zeit vor Hitler, in die Stimmung damals. Ich war kein Nazi. Aber ich gehörte auch nicht zu den Leuten, die es sich so leichtmachten und sagten, den Hitler muß man nicht ernst nehmen ". Zu einer wichtigen »Urteilsdifferenz« zwischen Vater und Sohn vgl. ebd., S. 281: »Über Hitler hat er [Ernst von Weizsäcker, Staatsekretär im Auswärtigen Amt 1938-1943] im Familienkreis zuerst im Ton der Verachtung, dann des Zorns geredet. Von 1936 erinnere ich mich positiv, daß er Hitler als 'Narr und Verbrecher < bezeichnete. 'Narr hat er wohl immer gesagt, >Verbrecher mag seit dem 30. Juni 1934 dazugekommen sein. Im ersten Halbjahr 1933 hatte ich nicht einen Streit, aber eine Urteilsdifferenz mit ihm. Ich fand, er unterschätze Hitler. Ich hatte Hitler vor 1933 nur verachtet. 1933 entdeckte ich die Kraft der Bewegung, das, was Wilhelm Kütemeyer später >die Pseudoausgießung des Heiligen Geistes von 1933 « nannte. Mein Vater und ich hatten wohl beide partiell recht, partiell unrecht. Er sah das Absurd-Verbrecherische, aber nicht das Format. Ich sah das Format. Die Bewegung blieb mir in Verhaltensweisen und Ansichten fremd, aber ich geriet in Versuchung, Einfluß auf ihre Führung zu suchen - naive Selbstüberschätzung «.

136 Ebd., S. 364 f.

137 Ebd., S. 365. 
für von Weizsäcker war, um in der sanften Bundesrepublik beim Namen genannt zu werden.

Zum Verständnis der von-Weizsäcker-Sprache erscheint es sinnvoll, das intime Verständnis eines atomaren peers, das der deutsche Physiker durch sein intensives Studium der "Sache Oppenheimer " gewonnen hatte, auf ihn selbst anzuwenden. ${ }^{138}$ Von Weizsäcker verstand, dass Oppenheimer » eine komplizierte persönliche Motivation Leuten erklären muß, von denen er weiß, daß sie glauben, er lügt, wenn er die Wahrheit sagt. Nur wenn er lügt, kann er so reden, daß sie sich einbilden, er sage die Wahrheit «. ${ }^{139}$ Mir scheint, von Weizsäcker stand vor demselben Problem wie Oppenheimer. Das ist eine Einladung an den Historiker, von Weizsäckers historische Angaben ebenfalls als ein Gemisch aus Verheimlichung und Offenheit zu entschlüsseln. Was also glaubhaft wirkt, könnte auf falsche Wege führen, und was unglaublich erscheint, könnte die Wahrheit sein. Von Weizsäckers Sprechweise war auf eine bestimmte Weise verschlungen; er deutete etwas Wahres an, sprach es sogar gelegentlich aus, beließ aber den leicht zu beindruckenden »Leuten « die falschen oder vereinfachten Vorstellungen, die sie sich über nicht näher erläuterte Dinge machten, wenn ihm die volle Erklärung Schwierigkeiten bereitet hätte und auf Unverständnis gestoßen wäre.

Die Anwendung dieser Interpretationsmethode auf den Friedensplan von Weizsäckers muss den bekannten »Friedensforscher " ausklammern, der den von Weizsäcker'schen Lernprozess voraussetzt. Der weise von Weizsäcker stand dem jungen von Weizsäcker nicht zur Verfügung. Der von Weizsäcker, der zu Hitler vordringen wollte, war eine Gestalt, die mit dem späteren von Weizsäcker, der zum Pazifismus der Bundesrepublik beitrug, nichts zu tun hatte. Auf dem Höhepunkt Hitler'scher Machtentfaltung konnte Frieden nur eine Bedeutung haben: Pax Germanica. Und ein solcher Siegfrieden war kein erschwerender Umstand, sondern der Ausgangspunkt im Denken des jungen von Weizsäcker. Der junge von Weizsäcker dachte nicht daran, Hitler einen Vortrag darüber zu halten, »dass er nunmehr eine Politik machen muss, die die Menschheit nicht umbringt, sondern die die Institution des Krieges abschafft « ${ }^{140}$ Das ist eine von Weizsäcker'sche Redeweise à la Oppenheimer (er lügt, damit er so reden kann, dass wir uns einbilden, er sage die Wahrheit). Der von Weizsäcker, den ich kannte, war der geläuterte von Weizsäcker, der uns die nackte Wahrheit vorenthielt und nach der Wende 1945 mit der humanistischen Idee hervortrat, dass man das Kriegführen beenden müsse, da Atomwaffen den

138 Siehe ebd., S. 370: »Ich habe nur zweimal mit ihm [Oppenheimer] geredet, aber ich habe das ganze Oppenheimer-Hearing studiert «. Vgl. die Verhandlungsprotokolle »In der Sache J. Robert Oppenheimer « (über eintausend kleingedruckte Seiten): U.S. Atomic Energy Commission 1954 a; U.S. Atomic Energy Commission 1954 b.

139 Weizsäcker 1988, S. 370.

140 Von Weizsäcker im Interview mit Schaaf, Spitzer 2006, S. 7.

Leviathan, 41. Jg., 3/2013 
Selbstmord der Menschheit auslösen können. ${ }^{141}$ Der junge von Weizsäcker hingegen, der bis 1945 in einem Staat vorankommen wollte, der nicht Frieden suchte, sondern ab 1939 einen Angriffskrieg nach dem anderen begann, blieb ein uns Unbekannter.

Dass von Weizsäcker in der Zeit seiner Plutoniumpläne nichts anderes im Sinn hatte, als Hitler "gerade noch " zu retten, ist eine anachronistische Aussage, das heißt eine Formulierung, die nur im Nachhinein möglich war. Im »gerade noch « drückte sich der ältere von Weizsäcker aus, der den Untergang des Dritten Reichs miterlebt hatte und wusste, was die Zukunft für Hitlers russischen Blitzkrieg bereithielt. ${ }^{142}$ Der junge von Weizsäcker war kein Hellseher; er erwartete den deutschen Sieg und wollte Hitler die Überwaffe anbieten, um die Vormachtstellung Deutschlands zu garantieren, nicht um den Selbstmord des Diktators und die Verwüstung des Landes zu verhindern. Was von Weizsäcker 1941 voraussah, war Deutschlands Aufstieg zur ersten Atommacht in der Welt. Darauf, dachte er, würde der "hochbegabte « Hitler anspringen. Die Macht der Atombombe sollte es dem über Westeuropa triumphierenden Diktator ermöglichen, die "Angelsachsen « in Schach zu halten (USA) beziehungsweise in die Knie zu zwingen (Großbritannien) und Russland zu kolonisieren. Die »Politik des Friedens «, über die der junge von Weizsäcker mit Hitler reden wollte, betraf, das dürfen wir annehmen, die europäische Neuordnung unter deutscher Vorherrschaft. Private Quellen (Briefe und Tagebücher) wären hier zur Bestätigung, Widerlegung oder Modifizierung dieser begründeten Vermutung hilfreich.

Alles, was über die Aktivitäten des jungen von Weizsäcker bekannt ist, spricht dafür, dass er zwischen 1940 und 1941 den Bau einer Plutoniumbombe nicht nur befürwortete und anstrebte, sondern zu forcieren und mit seinem Namen zu verbinden suchte. Von Weizsäckers »Patentanmeldung « belegt sein persönliches und sachliches Interesse. Auf dem Titelblatt des Dokuments heißt es: »Für die in der Anlage überreichte Patentanmeldung ist als Erfinder dem Reichspatentamt gegen-

141 Von Weizsäcker konnte sich sehr klar ausdrücken. Seine Atomwaffen-und-Krieg-Formulierung, die ich als wissenschaftlicher Mitarbeiter am Starnberger Max-PlanckInstitut in den 1970er Jahren oft gehört habe, war von Anfang bis Ende prägnant und präzise. Siehe zum Beispiel Weizsäcker 1993, S. 341: »Wenn Atombomben möglich sind, wird es jemanden geben auf der Welt, der sie macht, ganz einerlei, was wir tun. Und wenn es diese Bombe geben wird, wird es jemanden geben, der sie einsetzt. Und dann bleibt der Menschheit nur die Alternative, entweder das Motiv, solche Bomben zu machen und sie einzusetzen, völlig zu eliminieren, daß heißt, den Krieg als Institution abzuschaffen - nicht die Bombe, sondern den Krieg -, oder sich selber zugrunde zu richten «.

142 Der junge von Weizsäcker nahm, wie es scheint, den geplanten Russlandfeldzug ohne große Besorgnis zur Kenntnis: Staatssekretär von Weizsäcker hielt den Krieg gegen Russland, der am 22. Juni 1941 begann, »für ein Unglück «(Hill 1974, S. 248); sein Sohn Carl Friedrich, den er Anfang Mai 1941 einweihte, konnte den väterlichen »Standpunkt eigentlich nicht verstehen oder nicht billigen « (ebd., S. 254). 
über namhaft zu machen: Herr Dozent Dr. C.F. von Weizsäcker «. ${ }^{143}$ Auf Seite vier wird hervorgehoben: »Dieser Sprengstoff [Plutonium] würde an frei werdender Energie pro Gewichtseinheit jeden anderen 10 Millionen mal übertreffen und nur mit dem reinen $235 \mathrm{U}$ vergleichbar sein «. ${ }^{144}$ Die Nummer fünf der sechs »Patentansprüche« ist als Hauptgrund für von Weizsäckers restriktiven bundesrepublikanischen Umgang mit der historischen Wahrheit anzusehen; der fünfte Patentanspruch lautete:

»Verfahren zur explosiven Erzeugung von Energie und Neutronen aus der Spaltung des `Elements 94‘, dadurch gekennzeichnet, daß das nach Anspruch 3 hergestellte 'Element 94، in solcher Menge an einen Ort gebracht wird, z. B. in eine Bombe, daß die bei einer Spaltung entstehenden Neutronen in der überwiegenden Mehrzahl zur Anregung neuer Spaltungen verbraucht werden und nicht die Substanz verlassen «. ${ }^{145}$

Auf der »freien Straße zur Atombombe «, ${ }^{146}$ die Heisenberg im September 1941 vor dem deutschen Uranprojekt liegen sah, war von Weizsäcker mit seinem Patentbegehren (ohne ausdrückliche Zustimmung seiner Kollegen) vorgeprescht. Heisenbergs Uranverein und Diebners Forschungsgruppe zogen damals noch an einem Strang. Es scheint, dass von Weizsäcker die Plutoniumbombe zuerst auf dem Dienstweg zu lancieren versuchte. Ein »Kurzer Bericht über die eventuelle praktische Auswirkung der Uranuntersuchungen aufgrund einer Rücksprache mit Dr. Diebner « 147 deutet darauf hin.

In diesem Bericht erörterte von Weizsäcker verschiedene Anwendungsmöglichkeiten der Uranspaltung, unter anderem als Raketenantrieb, den er jedoch als eine »Sache zukünftiger Entwicklung « hintanstellte. Im Sommer 1941 kam es von Weizsäcker hauptsächlich auf zwei eng miteinander verbundene Verwendungsweisen an: »Vordringlich ist nach dem derzeitigen Stand der Versuche die Entwicklung [...] als Wärmemaschine und als Sprengstoff «. ${ }^{148}$ Nach einer mündlichen Anhörung war von Weizsäcker offenbar gebeten worden, seine Dringlichkeitseinschätzung zu Papier zu bringen, um Diebner etwas Schriftliches für die oberen Entscheidungsträger des HWA an die Hand zu geben. Als danach nichts geschah, sah sich von Weizsäcker nach einem machtvolleren Partner um. Das kann aus einer Bemerkung im Spiegel-Interview von 1991 geschlossen werden, worin von Weizsäcker ein Gespräch mit Schumann erwähnte und dass er ihn gefragt habe, »ob man höhere Regierungsstellen über unsere Arbeit noch weitergehend informieren sollte oder

143 Karlsch 2005, S. 322. Das Patent wurde von Karlsch in Moskau aufgefunden und teilweise in Hitlers Bombe publiziert. Es befindet sich jetzt im Archiv der MPG (siehe Anmerkung 86). Das Dokument ist nicht datiert und nach Karlsch "wahrscheinlich [vom] Frühsommer 1941 « (ebd., S. 321); es trägt den Titel: » Energieerzeugung aus dem Uranisotop der Masse 238 und anderen schweren Elementen (Herstellung und Verwendung des Elements 94) «.

144 Ebd., S. 323.

145 Ebd., S. 323 f.

146 Irving 1967 b, S. 106.

147 Archiv der Max-Planck-Gesellschaft, I. Abt., Rep. 34 KWI für Physik, Nr. 93/2.

148 Walker 2007, S. 362. 
nicht «. ${ }^{149}$ Als sich Diebners Chef dagegen aussprach, hatte von Weizsäcker das Ende des Dienstwegs erreicht. Wie konnte seine »Waffe« nun doch noch höchste Priorität erhalten? Hitlers Unterstützung war unter den gegebenen Umständen die letzte Möglichkeit, die Plutoniumbombe für Deutschland zu realisieren. Die Idee, von Weizsäckers Wissen mit dem »Instrument der Vorsehung « zu paaren, um damit die deutsche Beherrschung Europas zu zementieren, war insofern allenfalls naivmachiavellistisch, aber nicht »verrückt «.

Von Weizsäckers träumerischer Versuch, das deutsche Uranprojekt auf die »explosive« Anwendung des Elements 94 auszurichten, blieb im polykratischen Gestrüpp der »Unterlinge « hängen; der Versuch war nicht mit Heisenberg abgestimmt, und es fand sich kein starker »Wissenschaftsbevollmächtigter ", der willens und fähig gewesen wäre, eine strategische Entscheidung dieser Tragweite zu befürworten und durchzusetzen. Ob von Weizsäcker konkrete Anstrengungen unternahm, eine Audienz zum Plutoniumvortrag im Führerhauptquartier zu erhalten, ist nicht bekannt, aber auch unerheblich für die Feststellung, dass er in den beiden ersten Kriegsjahren die militärische Ausrichtung des deutschen Projekts und insbesondere den Bau einer Plutoniumbombe anstrebte. Die Ironie der »UranbombenGeschichte" wollte es, dass um dieselbe Zeit, als sich die deutsche Entscheidung anbahnte, das Uranprojekt zurückzuschrauben, die Vereinigten Staaten beschlossen, sowohl die Uranbombe (Little Boy, Hiroshima) als auch die Plutoniumbombe (Fat Man, Nagasaki) mit vollem Einsatz aller verfügbaren Kräfte zu verfolgen. ${ }^{150}$ Im Winter 1941/42 wusste keine Seite von der gegensätzlichen Entscheidung der anderen.

\section{Schluss: Glücklich in Straßburg »durchgesetzt»}

»Ich finde es ja für Weizsäcker lehrreich, das Intrigenspiel mal am eigenen Leib auszuprobieren, aber ich wünschte doch sehr, daß er sich durchsetzen kann «. 151

Werner Heisenberg, 19. Juli 1941

Für von Weizsäcker wurde die Plutoniumbombe im Sommer 1941 durch ein Karriereproblem in den Hintergrund gedrängt. Von Weizsäcker, der 1936 bei Heisenberg habilitiert hatte, war seit Anfang 1941 im Gespräch, an die »Reichsuniversität

149 Weizsäcker 1991 b, S. 235. Einer brieflichen Angabe von 1987 zufolge sprach von Weizsäcker zweimal mit Schumann, das erste Mal im September 1939 und das zweite Mal »später «; siehe Weizsäcker, Hora 2002, S. 212: »Mit Schumann habe ich - wenn ich mich recht erinnere - später nur noch einmal gesprochen in einer Angelegenheit [Plutoniumbombe], die ich jetzt nicht ausführen will, aus der auch nichts geworden ist «.

150 Siehe Walker 1990 a, S. 68.

151 Hirsch-Heisenberg 2011, S. 181. 
Straßburg « (RUS) berufen zu werden, ${ }^{152}$ deren Gründung sofort nach der Niederlage Frankreichs anvisiert worden war. Im Elsass hatte schon einmal eine deutsche »Frontuniversität « ${ }^{153}$ bestanden, die Kaiser-Wilhelm-Universität (1872-1918), und an ihr hatte auch ein Weizsäcker von Anbeginn gelehrt, Julius Weizsäcker, ein Ranke-Schüler und Urgroßonkel. ${ }^{154}$ Nun ging es darum, die RUS im Sinne des Reichsministers für Wissenschaft, Erziehung und Volksbildung, Bernhard Rust, als »idealtypische nationalsozialistische Hochschule « zu etablieren und in ihr »Grenzlandpolitik, Hochschulreform und völkisch-rassische Fundierung « zu einer neuen Einheit zu verbinden. ${ }^{155}$ Mit dieser Aufgabe wurde der Historiker Ernst Anrich als Bevollmächtigter des Reichsdozentenführers beauftragt. ${ }^{156}$ Anrich setzte sich im Vorfeld und später als Dekan der Philosophischen Fakultät energisch dafür ein, mit der RUS nicht nur »die wissenschaftlichen Fragestellungen, die die deutsche Universität aus der nationalsozialistischen Weltanschauung schon entfaltet hat, dem Elsaß darzubringen «, sondern auch die Überwindung Frankreichs und seines »westlerischen Geistes « durch die »Ausstrahlung der deutschen europäischen Ordnungsidee über die Grenze hinüber in den Westen selber « 157 zu konsolidieren und mit rassistischer "Westforschung « zu legitimieren. ${ }^{158}$

Der Gründungsrektor der RUS, Karl Schmidt, versicherte, alles zu tun, »daß diese Universität in ihrem ganzen Umfang, also auch mit jedem Einzelmann des Lehrkörpers, geschlossen für den Nationalsozialismus eingesetzt werden kann und sich selbst kämpferisch einsetzen wird «. ${ }^{159}$ Vor dem Hintergrund solcher Erwartungen war von Weizsäcker ein problematischer Kandidat. Er gehörte nicht der Partei an

152 Siehe Kant 1997, S. 17, Anmerkung 80. Eine weniger ausführliche Fassung dieses Preprints ist im Druck erschienen; siehe Kant 2005.

153 Inoffiziell wurde die Reichsuniversität Straßburg, die von 1941 bis 1944 bestand, als »NS-Kampfuniversität Straßburg « bezeichnet. Weitere NS-» Grenzlanduniversitäten « waren die Reichsuniversitäten Posen (1941-1945) und Prag (1939-1945). In Prag wirkte der sudetendeutsche Jurist und Rechtshistoriker Wilhelm Weizsäcker (1886-1961), ein Spross der bürgerlichen Linie des Weizsäcker-Geschlechts.

154 Siehe Wein 1989, S. 438.

155 Hausmann 2010, S. 194. Die RUS wurde am 23. November 1941 eröffnet.

156 Siehe Kettenacker 2005.

157 Hausmann 2010, S. 201 f. Anrich, der nach 1945 »seinen Idealen treu « blieb, gründete die Wissenschaftliche Buchgesellschaft Darmstadt und wechselte von der CDU zur NPD.

158 Ebd., S. 220 f. Hausmann hat die kurze, aber reiche Geschichte der RUS als "paradigmatisch « für die NS-Wissenschaftsplanung und -konzeption beschrieben und darauf hingewiesen, dass »das polykratische Gefüge des NS-Staates, in dem sich nicht nur das REM, sondern auch die Parteikanzlei, das Amt Rosenberg, die SS, das Auswärtige Amt, das Innen- und das Finanzministerium für die Universitäten und Hochschulen interessierten «, neue Handlungsspielräume eröffnete und Organisationsformen ermöglichte; siehe ebd., S. 229.

159 Zitiert nach Kant 1997, S. 4. Schmidt, ein Ophthalmologe, war zuvor Universitätsrektor in Bonn gewesen (1933-1939). 
und war kein Kämpfer, der sich offenkundig »in den Dienst und unter die Weltanschauung « 160 des real existierenden Nationalsozialismus gestellt hatte. Mit seinem »utopischen Nationalsozialismus «161 war er nicht öffentlich hervorgetreten. Sub specie aeternitatis bedeutete das jedoch nicht, dass von Weizsäcker im Hinblick auf eine längerfristige Entwicklung des Nationalsozialismus (die zumindest bis 1942 allgemein erwartet wurde) keine vorzügliche Wahl war. Seine fachliche Qualifikation stand zudem immer außer Frage - mit einer wichtigen, ironischen Einschränkung: Man musste, wie der künftige Straßburger Physikerkollege Wolfgang Finkelnburg, der der NSDAP angehörte, auf Seiten der modernen theoretischen (»jüdischen «) Physik Einsteins und Bohrs stehen. Für die »Deutsche Physik «162 von Philipp Lenard und Johannes Stark war von Weizsäcker natürlich ideologisch disqualifiziert, denn er, Heisenberg und andere hatten (nach groben, persönlichen Angriffen im Völkischen Beobachter und im Schwarzen Korps) die moderne Physik mit Erfolg verteidigt. Sie hatten die Mehrzahl aller Lehrstuhlinhaber auf ihre Seite gebracht und nach und nach auch Unterstützung in der Wehrmacht, Industrie, Regierung und Partei gewonnen. Und dafür mussten sie büßen. ${ }^{163}$

1939 wurde die Berufung Heisenbergs nach München (auf den Lehrstuhl seines Lehrers Arnold Sommerfeld) durch die NSDAP verhindert, ${ }^{164}$ und 1941, als von Weizsäckers Erstberufung an die RUS in Aussicht stand, erhob sich von Seiten der Partei auch gegen ihn im REM, der nominell entscheidenden Staatsbehörde, gezielter ideologischer Widerspruch. Nach dem Hauch von Schadenfreude, die im Motto dieses Abschnitts anklingt und Heisenbergs kritische Einschätzung der von Weizsäcker'schen Lust an den »kleinen taktischen Geschichten « erkennen lässt, informierte Heisenberg seine Frau im Sommer 1941 über den Stand des politischen »Intrigenspiels «:

»Weizsäckers Aussichten scheinen schlecht zu stehen. Er hat zwar sowohl von Rust wie von Mentzel gehört, er solle nach Strassburg gehen; dagegen hat der Sachbearbeiter Dr. Führer

160 Ebd.

161 Siehe Kapitel 4, S. 250.

162 Zur »deutschen « beziehungsweise "arischen « Physik siehe Richter 1980; Beyerchen 1982, S. 207-227; Walker 1990 a, S. 79-85.

163 Siehe Finkelnburg, zitiert in Hentschel 1996, S. 343: "The appointment of Messrs. v. Weizsäcker and myself at Strasbourg University, which were at that time still undecided, were delayed for almost two years with open reference to our actions against 'German physics; and we were then also treated unfairly besides (associate professorships instead of the planned full professorships) «.

164 Nachdem sich Rudolf Heß, der Stellvertreter Hitlers, und Walter Schultze, der Führer des Reichsdozentenbundes, gegen die Berufung Heisenbergs ausgesprochen hatten, wurde Heisenberg darüber informiert, dass Himmler ihn zwar unterstützt habe, aber die Parteientscheidung gegen Heisenbergs Berufung nach München nicht umstoßen wollte. » Wenn Heisenberg jetzt doch einen Ruf nach München erhalte, würde die Partei unglaubwürdig werden. Himmler war mit Schultzes Ansicht nicht einverstanden, verspürte aber wenig Lust, sich wegen einer Professur mit der Partei anzulegen « (Walker 1990 a, S. 84). 
in einem Brief an die Universität Strassburg mitgeteilt, W's Kandidatur käme nicht in Frage $\ll \cdot{ }^{165}$

Wilhelm Führer war einer der beiden REM-Referenten für die Naturwissenschaften (der andere war Max Demmel). Als Parteimitglied (seit 1930), SS-Mann (seit 1933), promovierter Astronom, Vertreter der arischen Physik und mit Zuständigkeit im REM für Berufungen im Bereich der Physik (seit 1939) war er ein ernst zu nehmender Widersacher. ${ }^{166}$ Er hatte erfolgreich gegen Heisenberg agiert und widersprach nun den Wünschen des Gründungsdekans der Naturwissenschaftlichen Fakultät, Georg Niemeier, einem Geographen, der in Straßburg vier Physik-Lehrstühle zu besetzen hatte und darauf drang, von Weizsäcker mit der Wahrnehmung der theoretischen Physik zu betrauen. Dass Führer ungeachtet dessen und hochkarätiger Befürworter zum Trotz - Rust war immerhin der Leiter des REM und Rudolf Mentzel ein einflussreicher Multifunktionär des Regimes - gegen von Weizsäcker Front machte, spricht für ein Tauziehen zwischen Partei und Staat sowie die große geopolitisch-ideologische Bedeutung Straßburgs für Parteiaktivisten.

Die Intervention, die von Weizsäcker »durchsetzte «, ging von Ernst von Weizsäcker aus, von Weizsäckers Vater. ${ }^{167}$ Die Universität Straßburg hatte ihn um Hilfe gebeten. ${ }^{168}$ Er besorgte der REM-Führung die nötige Rückendeckung, um die Berufung des jungen von Weizsäcker gegen den eigenen Fachreferenten am Ende doch befürworten zu können. Im Herbst 1941 verlangte der Staatssekretär eine Überprüfung des Falls von der Parteikanzlei, dem zentralen Führungsorgan der NSDAP, indem er unter anderem hervorhob, dass sein Sohn dem Nationalsozialistischen Deutschen Dozentenbund (NSDDB) und der NS-Volkswohlfahrt angehöre. ${ }^{169}$ Der Vater hatte den Sohn zwar vom Parteieintritt abhalten können, nicht aber vom Eintritt in den NS-Lehrerbund (NSLB), in den er am 1. August 1934 aufgenommen wurde. ${ }^{170} \mathrm{Ob}$ von Weizsäcker auch in den NSDDB mit überwechselte, als der ein Jahr später aus der Reichsfachschaft Hochschullehrer des NS-Lehrerbunds hervorging, ist nicht geklärt, aber in Anbetracht der von Weizsäcker'schen Hochschul-

165 Hirsch-Heisenberg 2011, S. 180 f. Im Anschluss an seine Hoffnung, dass von Weizsäcker reüssieren möge, bekundete Heisenberg eine zentrale (und berechtigte, siehe Anmerkung 114) Angst der damaligen Zeit: »Ich fürchte, man wird dafür sorgen, dass er jetzt eingezogen wird, und dann kann man mit gutem Vorwand einen Herrn von den Qualitäten [Bruno] Thüring [Vertreter der arischen Physik] berufen« (ebd., S. 181).

166 Siehe Beyerchen 1982, S. 211, 242.

$167 \mathrm{Zu}$ Ernst von Weizsäcker und der politischen »Urteilsdifferenz « im Vater-Sohn-Verhältnis siehe Anmerkung 135.

168 Siehe Kelly 1973, S. 388: »Strasbourg, evidently in agreement with the Reich Ministry of Education, had appealed to Weizsäcker's father, the influential State Secretary in the Foreign Office, to intervene and to appeal to the Party Chancellery «.

169 Vgl. Walker 1990 a, S. 87.

170 Von Weizsäckers NSLB-Mitgliedsnummer war 311818: Bundesarchiv (ehemals BDC) NSLB. 
lehrerlaufbahn wahrscheinlich. ${ }^{171}$ Der amerikanische Historiker Reece Kelly fand den väterlichen Hinweis auf von Weizsäckers Mitgliedschaft im »Dozentenbund « so erstaunlich, dass er einen Fehler im Ausdruck vermutete. ${ }^{172}$

Ernst von Weizsäckers Demarche wurde sowohl von Schultzes NSDDB als auch von Alfred Rosenbergs Außenpolitischem Amt unterstützt, zwei Parteiorganisationen, die damals heftig miteinander konkurrierten. Das positive Votum des NSDDB kam von Gustav Borger, der seit 1938 das »Amt Wissenschaft « im NS-Dozentenbund leitete und im November 1940 eine Debatte über die »Deutsche Physik « moderiert hatte, die als » Münchener Religionsgespräch « bekannt wurde. Dass von Weizsäcker in diesem Streitgespräch die Relativitätstheorie mit Erfolg vertrat und an der gemeinsamen Fünf-Punkte-Erklärung mitgewirkt hatte, die zur »Anerkennung der Relativitätstheorie und der Quantenmechanik durch eine Parteistelle «173 führte, hatte Borger nicht vergessen. Am 17. November 1941 erklärte er in einem Schreiben an die Parteikanzlei, von Weizsäcker gehöre als Mensch wie als Wissenschaftler ohne Zweifel zu den Allerfähigsten der jüngeren Generation und verdiene, nach Straßburg berufen zu werden. ${ }^{174}$ Das Amt Rosenberg machte für von Weizsäcker ebenfalls eine Ausnahme, indem es wenig später, am 28. November, die Position bezog, dass an deutschen Universitäten auch der rein akademische Wissenschaftlertyp ausreichend Platz finden müsse. ${ }^{175}$ Das Straßburger Interesse an von Weizsäcker vertrat Niemeier, der die »wehrhafte Bedeutung naturwissenschaftlicher Grundlagenforschung " erkannt hatte und im Hinblick auf die Nuklearphysik hervorhob:

»Die deutsche Sorge muß sein, [...] daß wir den Vorsprung, der durch die erste grundsätzliche Lösung des Problems [Entdeckung der Kernspaltung] gegeben war, behalten bezw. wiedergewinnen. Geschieht das nicht, dann können sich noch viel schwerer wiegende Folgen ergeben wie in einem ähnlichen Fall im Weltkrieg [...] [amerikanische Entwicklung der

171 So nahm von Weizsäcker beispielsweise am »ersten deutschen Mathematikerlager « teil, das der NSDDB vom 1.-3. Juli 1938 in der Ützdorfer Jugendherberge nahe Bernau veranstaltete. Von Weizsäcker sprach sich auf diesem »Dozentenlager « für die moderne theoretische Physik aus; siehe Hentschel 1996, S. 340, Anmerkung 9.

172 Siehe Kelly 1973, S. 388, Anmerkung 38: »According to the Party Chancellery, the elder Weizsäcker had tried to refute the charge of his son's lack of political commitment by citing, surely mistakenly, the younger Weizsäcker's membership in the >Dozentenbund (he probably meant >Dozentenschaft and the NS-Volkswohlfahrt. The latter seems an innocuous enough gesture on the part of the young scientist, and his membership in the Dozentenschaft, if that was what was meant, was automatic. The only other 'political activity the elder Weizsäcker could cite was his son's volunteering for work service and military service ".

173 Beyerchen 1982, S. 241.

174 Kelly 1973, S. 388. Das Resultat der Debatte wurde in einem internen Memorandum des NSDDB im März 1941 dahingehend festgehalten, dass die Physik in Deutschland in zwei Lager zerfalle: »the smaller one around Lenard and a much larger one around Heisenberg [...] The group around Heisenberg, for example, has by far the most German chairs for physics in its possession and also is strongly placed in the war industry« (ebd., S. 387, Anmerkung 36).

175 Ebd., S. 389. 
Elektronenröhre]. Es könnte jetzt die Gefahr bestehen, daß uns die Amerikaner auf der Grundlage des deutschen Forschungsergebnisses, nämlich der Kettenreaktion bei der Kernumwandlung, in der praktischen Auswertung zuvorkommen, weil sie eine größere Zahl, zum Teil arbeitsfähiger, kostspieliger Spezialapparaturen besitzen, welche die erforderliche große Neutronenintensität erzeugen können. Solche entsprechenden Apparate sind in Deutschland in zu geringer Zahl vorhanden. Ihre Schaffung ist notwendig, weil mit ihnen kernphysikalische Grundprobleme einer Lösung zugeführt werden müssen, die vielleicht eines Tages über Sein oder Nichtsein von Völkern und Staaten entscheiden können «. ${ }^{176}$

Von Weizsäckers »Seilschaft « siegte, wenn auch nicht auf der ganzen Linie. Nach langem Zögern teilte das REM von Weizsäcker am 30. September 1942 mit, dass er nach Straßburg umziehen und dort ab 15. Oktober vertretungsweise den Lehrstuhl für theoretische Physik wahrnehmen solle. Am 11. März 1943 erhielt er schließlich rückwirkend zum 1. Januar die Ernennung zum Extraordinarius und Direktor des Instituts für theoretische Physik. ${ }^{177}$ Die Verzögerungen sowie der Umstand, dass ihm nur eine H2-Stelle zuerkannt wurde, waren den Parteikräften um Führer zu verdanken. Gleichwohl stellte die RUS-Berufung für von Weizsäcker einen großen persönlichen und für die Physiker-Gruppe um Heisenberg einen wichtigen institutionellen Erfolg in ihrem Kampf gegen die »Deutsche Physik « dar. Für den Uranverein war das kernphysikalische Interesse Niemeiers von Bedeutung, und die üppigen Mittel, die für die RUS bereitgestellt wurden, versprachen vorzügliche akademische Arbeitsbedingungen. ${ }^{178}$ Dass von Weizsäckers ununterbrochene Mitarbeit im geheimen Uranprojekt (von 1939 bis 1945 als »kriegswichtig « eingestuft) auch beim Berufungstauziehen half, war natürlich nicht offenkundig, ist aber anzunehmen. ${ }^{179}$ In den späten 1980er Jahren kommentierte von Weizsäcker die vielfältige Hilfe, ohne die seine glückliche Durchsetzung in den frühen 1940er Jahren nicht möglich gewesen wäre, mit einem Satz für die von Weizsäcker'sche Hermeneutik: »Ich habe nicht gewußt, daß ich so gut getarnt war «. ${ }^{180}$

Im Herbst 1944 beendete die heranrückende amerikanische Front von Weizsäckers kurze Lehr- und Forschungstätigkeit an der RUS. Als Goudsmit mit der AlsosMission in Straßburg eintraf, hatten sich von Weizsäcker und sein Assistent KarlHeinz Höcker mit Heisenbergs Hilfe schon über den Rhein ins oberschwäbische

176 Niemeier 1941, S. $733 \mathrm{f}$.

177 Siehe Kant 1997, S. 21.

178 Nach Hausmann investierte das Dritte Reich in die RUS während der drei Jahre ihrer Existenz »ein Vielfaches « von dem, was Frankreich zwischen den zwei Weltkriegen in die Université de Strasbourg investiert hatte; siehe Hausmann 2010, S. 217 f.

179 Siehe zum Beispiel den positiven NSDDB-Hinweis auf die Heisenberg-Gruppe im Zusammenhang mit der »war industry «; Fußnote 174.

180 Wein 1989, S. 437.

Leviathan, 41. Jg., 3/2013 
Hechingen abgesetzt. ${ }^{181}$ Die Papiere, die Goudsmit Ende November 1944 in den Straßburger Büros fand - »indiskrete Briefbögen « mit den Anschriften verschiedener Atomlabore, einschließlich der Adresse und Telefonnummer des nach Hechingen transferierten KWIP, private Korrespondenz und Berechnungen, die sich auf die Theorie einer Uranmaschine bezogen ${ }^{182}$-, ließen nicht nur auf eine hastige und/oder nachlässige Abreise schließen:

»We studied the papers by candlelight for two days and nights until our eyes began to hurt. Later, we took them back to Paris and analyzed them again in detail. Finally they were sent to Washington where they were once more analyzed, indexed, translated and interpreted. The conclusions were unmistakable. The evidence at hand proved definitely that Germany had no atom bomb and was not likely to have one in any reasonable time «. ${ }^{183}$

Ende April 1945 traf Goudsmits Spezialeinheit in Hechingen und Haigerloch ein. Einen Monat zuvor hatte dort noch ein letztes, vielversprechendes Experiment mit der Diebner'schen Reaktoranordnung stattgefunden. Aber auch das änderte nichts mehr an der generellen Richtigkeit der Alsos-Einschätzung, dass das deutsche Projekt 1945 weit von einer Atombombe entfernt war. In der Hoffnung, die Arbeiten irgendwie fortsetzen zu können, und von der Annahme beflügelt, einen großen Forschungsvorsprung gegenüber den Amerikanern herausgeholt zu haben, gab von Weizsäcker kurz vor dem Abtransport nach Farm Hall das übelriechende Versteck der geheimen deutschen Projektberichte preis: in einem Kanister » in der Abortgrube hinter seinem Hechinger Haus « ${ }^{184}$ Erst Hiroshima machte von Weizsäckers Vorsprungsannahme zunichte und rief den von Weizsäcker'schen Lernprozess hervor, der eine Zeitlang mit der irreführenden Deklarierung des deutschen Atomprojekts als » friedliche Entwicklung « Hand in Hand ging. Dass von Weizsäcker in der zweiten Hälfte des 20. Jahrhunderts ein hervorragender »Lehrer der Nation « war, ist unbestritten; dass er gleichzeitig den gesellschaftlichen Prozess der Aufklärung über die deutsche wie die eigene nukleare Vergangenheit behindert hat, ist allerdings auch nicht zu leugnen.

181 Heisenberg hatte den Generalsekretär der KWG, Ernst Telschow, im Oktober 1944 gebeten, von Weizsäcker und Höcker notaufnehmen zu dürfen; siehe Kant 1997, S. 35, Anmerkung 182: »Herr Prof. v. Weizsäcker [...], der auch als Professor an der Universität Strassburg an den Kriegsarbeiten des Instituts teilgenommen hat, muß auf Anordnung seiner vorgesetzten Behörde das Institut für theoretische Physik der Universität Strassburg an eine geeignete Stelle verlagern. Da dieses Institut nur aus ihm und einem Assistenten besteht, hat er mich gebeten, ihm einen Raum in unserer Verlagerungsstelle Hechingen zur Verfügung zu stellen. [...] da Herr v. Weizsäcker an den Arbeiten des Instituts über das Uranproblem sehr stark beteiligt ist, möchte ich diese Bitte erfüllen «.

182 Goudsmit 1947, S. 69 f.

183 Ebd., S. 70 f.

184 Wein 1989, S. 441. Siehe auch Goudsmit 1947, S. 108 f. Nachdem Goudsmit einen amerikanischen Leutnant damit beauftragt hatte, den Behälter aus dem outhouse von Weizsäckers herauszufischen, platzierte der Soldat his obnoxious find unter Goudsmits offenem Fenster. 


\section{Literatur}

Bagge, Erich; Diebner, Kurt; Jay, Kenneth 1957. Von der Uranspaltung bis Calder Hall. rowohlts deutsche enzyclopädie. Hamburg: Rowohlt.

Bernstein, Jeremy. Hrsg. 2001 [1996]. Hitler's uranium club: the secret recordings at Farm Hall. 2. Auflage. New York: Copernicus Books.

Bethe, Hans A. 1958. »Brighter than a thousand suns «, in Bulletin of the Atomic Scientists 14, 10, S. 426-428.

Beyerchen, Alan D. 1982. Wissenschaftler unter Hitler: Physiker im Dritten Reich. Frankfurt a. M.: Ullstein.

Blum, Walter; Dürr, Hans-Peter; Rechenberg, Helmut. Hrsg. 1989. Werner Heisenberg: Gesammelte Werke / Collected Works V: Wissenschaft und Politik. München, Zürich: Piper.

Bohr, Niels 2002. Release of documents relating to 1941 Bohr-Heisenberg meeting, Niels Bohr Archive. http://nba.nbi.dk/release.html (Zugriff vom 08.08.2013).

Brandt, Reinhard; Karlsch, Rainer 2007. "Kurt Starke und die Entdeckung des Elements 93. Wurde die Suche nach den Transuranen verzögert? ", in Für und Wider »Hitlers Bombe", hrsg. v. Karlsch, Rainer; Petermann, Heiko, S. 293-326. Münster: Waxmann.

Carson, Cathryn 2010. Heisenberg in the atomic age: science and the public sphere. Publications of the German Historical Institute. New York: Cambridge University Press.

Cassidy, David C. 1992. Uncertainty: the life and science of Werner Heisenberg. New York: W.H. Freeman.

Ciesla, Burghard 2007. »Das Heereswaffenamt und die KWG im >Dritten Reich schen Forschungsbeziehungen zwischen 1918 und 1945 ", in Gemeinschaftsforschung, Bevollmächtigte und der Wissenstransfer, hrsg. v. Maier, Helmut, S. 32-76. Göttingen: Wallstein.

Dönhoff, Marion Gräfin; Sommer, Theo 1992. »>Der Wandel des Bewußtseins ist unterwegs.< Die Verantwortung des Wissenschaftlers in unserer Zeit: Der Physiker und Philosoph Carl Friedrich von Weizsäcker wird in dieser Woche 80 Jahre alt. Ein Gespräch «, in Die Zeit, 26. Juni 1992, S. 9-10.

Dörries, Matthias. Hrsg. 2005. Michael Frayn's Copenhagen in debate: historical essays and documents on the 1941 meeting between Niels Bohr and Werner Heisenberg. Berkeley papers in history of science. Berkeley: Office for History of Science and Technology.

Frank, Charles. Hrsg. 1993. Operation Epsilon: the Farm Hall transcripts. Berkeley: University of California Press.

Frayn, Michael 2000. Copenhagen. New York: Anchor Books.

Goudsmit, Samuel Abraham 1947. Alsos. New York: Henry Schuman.

Groves, Leslie R. 1962. Now it can be told: the story of the Manhattan Project. New York: Harper.

Hausmann, Frank-Rutger 2010. »Wissenschaftsplanung und Wissenschaftslenkung an der Reichsuniversität Straßburg (1940-1944) «, in Selbstmobilisierung der Wissenschaft, hrsg. v. Dinçkal, Noyan; Dipper, Christoff; Mares, Detlev, S. 187-230. Darmstadt: Wissenschaftliche Buchgesellschaft.

Heisenberg, Werner 1946. »Über die Arbeiten zur technischen Ausnutzung der Atomkernenergie in Deutschland ", in Werner Heisenberg: Gesammelte Werke. Reihe V: Wissenschaft und Politik, hrsg. v. Blum, Walter; Dürr, Hans-Peter; Rechenberg, Helmut, S. 28-32. München, Zürich: Piper.

Heisenberg, Werner 1956/57. »Two letters to Robert Jungk (transcription and translation) «, in Homepage von Jochen H. Heisenberg. http://werner-heisenberg.physics.unh.edu/ (Zugriff vom 09.04.2013).

Heisenberg, Werner 1969. Der Teil und das Ganze. Gespräche im Umkreis der Atomphysik. München: Piper.

Hentschel, Klaus. Hrsg. 1996. Physics and National Socialism: an anthology of primary sources. Basel: Birkhäuser.

Hill, Leonidas E. Hrsg. 1974. Die [Ernst von] Weizsäcker-Papiere, 1933-1950. Frankfurt a. M., Berlin, Wien: Propyläen. 
Hirsch-Heisenberg, Anna Maria. Hrsg. 2011. »Meine liebe Li! Der Briefwechsel 1937-1946. Mit Fotos und bislang unveröffentlichten Tagebuchaufzeichnungen aus dem Privatarchiv. St. Pölten: Residenz.

Hoffmann, Dieter 1993 a. »Operation Epsilon«, in Operation Epsilon, hrsg. v. Hoffmann, Dieter, S. 9-59. Berlin: Rowohlt.

Hoffmann, Dieter. Hrsg. 1993 b. Operation Epsilon. Die Farm-Hall-Protokolle oder die Angst der Alliierten vor der deutschen Atombombe. Deutsch von Wilfried Sczepan. Berlin: Rowohlt.

Irving, David 1967 a. The virus house. London: William Kimber.

Irving, David 1967 b. Der Traum von der deutschen Atombombe. Gütersloh: Sigbert Mohn.

Irving, David 1967 c. ")So groß wie eine Ananas...< SPIEGEL-Serie über den Wettlauf um die Atombombe im Zweiten Weltkrieg: Die Deutschen lagen vorn«, in Der Spiegel 23-27, 29. Mai bis 26. Juni 1967.

Jensen, Hermann 1992. "Warum Hitler die Bombe nicht baute. Eine neue These zum Gespräch zwischen Bohr und Heisenberg 1941 und den welthistorischen Folgen «, in Die Zeit, 24. April 1992, S. 41-42.

Jungk, Robert 1956. Heller als tausend Sonnen: Das Schicksal der Atomforscher. Stuttgart: Scherz \& Goverts.

Jungk, Robert 1964. Heller als tausend Sonnen: Das Schicksal der Atomforscher. rororo Tachenbuch 6629/6630 (ungekürzte Ausgabe). Reinbek bei Hamburg: Rowohlt.

Jungk, Robert 1993. Trotzdem: Mein Leben für die Zukunft. München: Carl Hanser.

Kant, Horst 1993. »Werner Heisenberg und das Kaiser-Wilhelm-Institut für Physik in Berlin «, in Werner Heisenberg, hrsg. v. Geyer, Bodo; Herwig, Helge; Rechenberg, Helmut, S. 152-158. Heidelberg: Spektrum.

Kant, Horst 1997. Zur Geschichte der Physik an der Reichsuniversität Straßburg in der Zeit des Zweiten Weltkrieges. www.mpiwg-berlin.mpg.de/Preprints/P73.PDF (Zugriff vom 01.06.2013). Berlin: Max-Planck-Institut für Wissenschaftsgeschichte.

Kant, Horst 2005. »Betrachtungen zur Physik an der Reichsuniversität Straßburg 1942-1944 «, in Les Reichsuniversitäten de Strasbourg et de Poznan et les résistances universitaires, 1941-1944, hrsg. v. Baechler, Christian; Igersheim, François; Racine, Pierre, S. 185-203. Strasbourg: Presses Universitaires de Strasbourg.

Kant, Horst 2011. »Peter Debye als Direktor des Kaiser-Wilhem-Instituts für Physik in Berlin «, in "Fremde"Wissenschaftler im Dritten Reich, hrsg. v. Hoffmann, Dieter; Walker, Mark, S. 76-109. Göttingen: Wallstein.

Karlsch, Rainer 2005. Hitlers Bombe: Die geheime Geschichte der deutschen Kernwaffenversuche. München: Deutsche Verlags-Anstalt.

Karlsch, Rainer; Petermann, Heiko. Hrsg. 2007. Für und Wider »Hitlers Bombe«: Studien zur Atomforschung in Deutschland. Cottbuser Studien zur Geschichte von Technik, Arbeit und Umwelt. Münster et al.: Waxmann.

Karlsch, Rainer; Walker, Mark 2005. New light on Hitler's bomb. http://physicsworld.com/cws/ article/print/2005/jun/01/new-light-on-hitlers-bomb (Zugriff vom 18.05.2013). Bristol: Institute of Physics.

Kelly, Reece Conn 1973. National Socialism and German university teachers: the NSDAP's efforts to create a National Socialist professoriate and scholarship. Doctor of Philosophy, History. Seattle: University of Washington.

Kettenacker, Lothar 2005. »Ernst Anrich und die Reichsuniversität Straßburg ", in Les Reichsuniversitäten de Strasbourg et de Poznan et les résistances universitaires, 1941-1944, hrsg. v. Baechler, Christian; Igersheim, François; Racine, Pierre, S. 83-96. Strasbourg: Presses Universitaires de Strasbourg.

Kleint, Christian 1993. "Die Leipziger Uranmaschinen ", in Werner Heisenberg, hrsg. v. Geyer, Bodo; Herwig, Helge; Rechenberg, Helmut, S. 141-151. Heidelberg: Spektrum.

Kraus, Elisabeth 2001. Von der Uranspaltung zur Göttinger Erklärung: Otto Hahn, Werner Heisenberg, Carl Friedrich von Weizsäcker und die Verantwortung des Wissenschaftlers. Würzburg: Königshausen \& Neumann.

Menge, Wolfgang 1991. Ende der Unschuld: Die Deutschen und ibre Atombombe. Berlin: Volk und Welt. 
Müller, Wolfgang D. 1990. Geschichte der Kernenergie in der Bundesrepublik Deutschland: Anfänge und Weichenstellungen. Stuttgart: Schäffer.

Nagel, Günter 2002. Atomversuche in Deutschland: Geheime Uranarbeiten in Gottow, Oranienburg und Stadtilm. Zella-Mehlis, Meiningen: Heinrich-Jung.

Niemeier, Georg 1941. »Von der wehrhaften Bedeutung naturwissenschaftlicher Grundlagenforschung «, in Straßburger Monatshefte 5, 11, S. 721-734.

Rechenberg, Helmut 1992. "Nicht die Spur von Landesverrat", in Die Zeit, 5. Juni 1992, S. 84.

Richter, Steffen 1980. »Die >Deutsche Physik«", in Naturwissenschaft, Technik und NS-Ideologie, hrsg. v. Mehrtens, Herbert; Richter, Steffen, S. 116-141. Frankfurt a. M.: Suhrkamp.

Rispens, Sybe Izaak 2011. »Einstein und Debye ", in »Fremde "Wissenschaftler im Dritten Reich, hrsg. v. Hoffmann, Dieter; Walker, Mark, S. 53-75. Göttingen: Wallstein.

Schaaf, Michael; Spitzer, Hartwig 2006. »Gespräch mit Carl Friedrich von Weizsäcker [7. März 1996] ", in Physik in unserer Zeit 39. www.wiley-vch.de/berlin/journals/phiuz/07-04/Weizsaecker-Interview-Weblayout.pdf (Zugriff vom 26.05.2013).

Schäfer, Wolf 2013. "Der sutopische< Nationalsozialismus: Ein gemeinsamer Fluchtpunkt im Denken von Martin Heidegger und Carl Friedrich von Weizsäcker? ", in Acta Historica Leopoldina 64, hrsg. v. Hentschel, Klaus; Hoffmann, Dieter (im Erscheinen).

Schwartz, Stephan 2011. "Naturwissenschaft und Technik im besetzten Dänemark: Das NielsBohr-Institut und sein Umfeld «, in »Fremde" Wissenschaftler im Dritten Reich, hrsg. v. Hoffmann, Dieter; Walker, Mark, S. 408-437. Göttingen: Wallstein.

Sime, Ruth Lewin 2007. » $)$ Die Uranspaltung hat da die ganze Situation gerettet<. Otto Hahn und das Kaiser-Wilhelm-Institut für Chemie während des Zweiten Weltkriegs ", in Gemeinschaftsforschung, Bevollmächtigte und der Wissenstransfer, hrsg. v. Maier, Helmut, S. 268-304. Göttingen: Wallstein.

Smyth, Henry De Wolf 1945. Atomic energy for military purposes. The official report on the development of the atomic bomb under the auspices of the United States government, 1940-1945. Princeton: Princeton University Press.

SPIEGEL 1957. »Und Führe Uns Nicht In Versuchung. Vom gespaltenen Atom zum gespaltenen Gewissen: die Geschichte einer menschheitsgefährdenden Waffe«, in Der Spiegel 19-25, 8. Mai bis 19. Juni 1957.

SPIEGEL 1962. »Am Tag, als die Bombe fiel: Versteckte Mikrophone belauschten deutsche Atomforscher «, in Der Spiegel 34, 22. August 1962, S. 46-47.

SPIEGEL 1988. »Übers Plutonium kann man was machen. ‘ Die Bemühungen deutscher Physiker im Zweiten Weltkrieg, eine Atombombe zu bauen «, in Der Spiegel 52, 26. Dezember 1988, S. $152-161$.

U.S. Atomic Energy Commission 1954 a. In the matter of J. Robert Oppenheimer. Transcript of hearing before Personnel Security Board. Washington: U.S. Government Printing Office.

U.S. Atomic Energy Commission Commission 1954 b. In the matter of J. Robert Oppenheimer. Texts of principal documents and letters of Personnel Security Board, general manager, commissioners. Washington: U.S. Government Printing.

Walker, Mark 1989. German National Socialism and the quest for nuclear power, 1939-1949. Cambridge, New York: Cambridge University Press.

Walker, Mark 1990 a. Die Uranmaschine: Mythos und Wirklichkeit der deutschen Atombombe. Vorwort Robert Jungk. Berlin: Siedler.

Walker, Mark 1990 b. »Heisenberg, Goudsmit and the German atomic bomb. Contrary to accounts based on Heisenbergs claims, the German fission research effort in World War II was indeed a nuclear weapons program, and contrary to Goudsmit's interpretations, the German team knew what it was doing ", in Physics Today 43, 1, S. 52-60.

Walker, Mark 2007. "Eine Waffenschmiede? Kernwaffen- und Reaktorforschung am KaiserWilhelm-Institut für Physik ", in Gemeinschaftsforschung, Bevollmächtigte und der Wissenstransfer, hrsg. v. Maier, Helmut, S. 352-394. Göttingen: Wallstein.

Wein, Martin 1989. Die Weizsäckers: Geschichte einer deutschen Familie. Stuttgart: Deutsche Verlags-Anstalt.

Weizsäcker, Carl Friedrich von 1940. Eine Möglichkeit der Energiegewinnung aus $238 U$. www.deutsches-museum.de/archiv/archiv-online/geheimdokumente/forschungszentren/ wien-heidelberg-strassburg/weizsaecker-energie-aus-u238/ (Zugriff vom 19.05.2013). München: Deutsches Museum. 
Weizsäcker, Carl Friedrich von 1954 [1948]. Die Geschichte der Natur. 2. Auflage. Die kleine Vandenhoeck-Reihe, Band 1. Göttingen: Vandenhoeck \& Ruprecht.

Weizsäcker, Carl Friedrich von 1977. Der Garten des Menschlichen: Beiträge zur geschichtlichen Anthropologie. München, Wien: Hanser.

Weizsäcker, Carl Friedrich von 1981. Der bedrohte Friede: Politische Aufätze 1945-1981. München, Wien: Hanser.

Weizsäcker, Carl Friedrich von 1988. Bewußtseinswandel. München, Wien: Hanser.

Weizsäcker, Carl Friedrich von 1991 a. »Die Bombe war zu teuer ", in Die Zeit, 19. April 1991, S. 83.

Weizsäcker, Carl Friedrich von 1991 b. » Ich gebe zu, ich war verrückt.< Carl Friedrich von Weizsäcker über die Chance der Deutschen im Zweiten Weltkrieg, die Atombombe zu bauen «, in Der Spiegel 17, 22. April 1991, S. 227-238.

Weizsäcker, Carl Friedrich von 1993. „Farm Hall und das deutsche Uranprojekt. Ein Gespräch mit Dieter Hoffmann, Helmut Rechenberg und Tilman Spengler ", in Operation Epsilon, hrsg. v. Hoffmann, Dieter, S. 331-360. Berlin: Rowohlt.

Weizsäcker, Carl Friedrich von et al. 1957. Text des Göttinger Manifests der Göttinger 18. www.uni-goettingen.de/de/54320.html (Zugriff vom 09.03.2013). Göttingen: GeorgAugust-Universität.

Weizsäcker, Carl Friedrich von et al. 1971. Kriegsfolgen und Kriegsverhütung. München, Wien: Hanser.

Weizsäcker, Carl Friedrich von; Hora, Eginhard 2002. Lieber Freund! Lieber Gegner! Briefe aus fünf Jahrzehnten. München, Wien: Hanser.

Weizsäcker, Viktor von 1973 [1940]. Der Gestaltkreis. Frankfurt a. M.: Suhrkamp.

Wirtz, Karl 1988. Im Umkreis der Physik. Karlsruhe: Kernforschungszentrum Karlsruhe. 
Zusammenfassung: Carl Friedrich von Weizsäcker trug sowohl zur Militarisierung als auch zur Entmilitarisierung der Atomkraft bei, und den Schock von Hiroshima beantwortete er mit einem historischen Mythos, der Legende von der »friedlichen « Ausrichtung des deutschen Atomprojekts unter Hitler. Von Weizsäcker vermochte es nicht, diese »dunkle Vergangenheit " offenzulegen, obwohl das Nachdenken über seine anfänglichen Atomwaffen-Ambitionen den historischen Lernprozess auslöste, der ihn 1957 zur Göttinger Erklärung befähigte. Indem er den jungen von Weizsäcker mitsamt der historischen Wahrheit über 50 Jahre lang fest unter Verschluss hielt, wurde der ältere von Weizsäcker nicht nur ein »Lehrer der Nation ", sondern auch ein Bremser des gesellschaftlichen Lernprozesses, der sich einredete, die Nachgeborenen könnten und würden das erste Kapitel der deutschen atomaren Geschichte sowieso nie »wirklich« verstehen.

Stichworte: Atombombe, Farm Hall, Forschungsgruppe Diebner, Göttinger Erklärung, Heereswaffenamt, historischer Lernprozess, Reichsuniversität Straßburg, Uranmaschine, Uranverein, Weizsäcker-Sprache

\title{
Plutonium bomb and civilian nuclear power: Carl Friedrich von Weizsäcker's contributions to the Third Reich and the Federal Republic
}

Summary: In his youth Carl Friedrich von Weizsäcker contributed to the militarization, in later years to the demilitarization, of nuclear power; he responded to the shock of Hiroshima with a historical myth. The legend of the " peaceful " direction of the German nuclear power project under Hitler was as Weizsäckerian as the attempt to provide Germany with the plutonium bomb in 1941. Von Weizsäcker was not able to expose this >dark past<, although contemplation of his initial nuclear weapon ambitions motivated the historical learning process that enabled him to devise the Göttingen Manifesto in 1957. By keeping both the young von Weizsäcker as well as the historical truth under tight wraps for over fifty years, the older von Weizsäcker became not only a steacher of the nation`, but also a brakeman of the societal learning process; he was convinced that later generations could and never would >really< understand the first chapter of German atomic history anyway.

Keywords: atom bomb, Farm Hall, Research Group Diebner, Göttingen Manifesto, Army Ordnance Office, historical learning process, Reich University of Strassburg, uranium machine, Uranium Club, Weizsäcker language

\author{
Autor \\ Prof. Dr. Wolf Schäfer \\ Direktor des Institute for Global Studies \\ Assoc. Dean, International Academic Programs \\ Stony Brook University \\ 100 Nicolls Road \\ Stony Brook, N.Y. 11794 \\ USA \\ wolf.schafer@stonybrook.edu
}

Leviathan, 41. Jg., 3/2013 